\title{
AN EFFECT SYSTEM FOR ALGEBRAIC EFFECTS AND HANDLERS*
}

\author{
ANDREJ BAUER AND MATIJA PRETNAR
}

Faculty of Mathematics and Physics, University of Ljubljana, Slovenia e-mail address: Andrej.Bauer@andrej.com, matija.pretnar@fmf.uni-lj.si

\begin{abstract}
We present an effect system for core Eff, a simplified variant of Eff, which is an ML-style programming language with first-class algebraic effects and handlers. We define an expressive effect system and prove safety of operational semantics with respect to it. Then we give a domain-theoretic denotational semantics of core Eff, using Pitts's theory of minimal invariant relations, and prove it adequate. We use this fact to develop tools for finding useful contextual equivalences, including an induction principle. To demonstrate their usefulness, we use these tools to derive the usual equations for mutable state, including a general commutativity law for computations using non-interfering references. We have formalized the effect system, the operational semantics, and the safety theorem in Twelf.
\end{abstract}

\section{INTRODUCTION}

An effect system supplements a traditional type system for a programming language with information about which computational effects may, will, or will not happen when a piece of code is executed. A well designed and solidly implemented effect system helps programmers understand source code, find mistakes, as well as safely rearrange, optimize, and parallelize code [11, 8. As many before us [11, 24, 25, 7] we take on the task of striking just the right balance between simplicity and expressiveness by devising an effect system for Eff [2], an ML-style programming language with first-class algebraic effects [17, 15] and handlers [19.

Our effect system is descriptive in the sense that it provides information about possible computational effects but it does not prescribe them. In contrast, Haskell's monads prescribe the possible effects by wrapping types into computational monads. In the implementation we envision effect inference which never fails, although in some cases it may be uninformative. Of course, typing errors are still errors.

An important feature of our effect system is non-monotonicity: it detects the fact that a handler removes some effects. For instance, a piece of code which uses mutable state is determined to actually be pure when wrapped by a handler that handles away lookups and updates.

2012 ACM CCS: [Software and its engineering]: Software notations and tools - General programming languages - Language features; [Theory of computation]: Semantics and reasoning.

Key words and phrases: algebraic effects, effect handlers, effect system.

* A preliminary version of this work was presented at CALCO 2013, see 3 . 
Our contributions are as follows:

(1) We define core Eff, a fragment of the language which retains the essential features of Eff, including first-class handlers and instances (Section 2), although we leave out dynamic creation of new instances.

(2) We give small-step and big-step operational semantics for core Eff and show them to be equivalent (Section 3).

(3) We devise an expressive effect system for core Eff and prove safety of the operational semantics with respect to it (Section 4).

(4) Using the standard domain-theoretic apparatus and Pitts's theory of minimal invariant relations [14, we provide denotational semantics for core Eff and prove an adequacy theorem (Section 5 ).

(5) We identify a set of observational equivalences and an induction principle that allow us to reason about effectful computations (Section 6).

(6) We demonstrate how the equivalences are used by deriving the standard equations for state from general principles. The induction principle is used in a proof of a general commutativity law which allows us to interchange two computations that use non-interfering references (Section 7).

(7) We formalized core Eff, the operational semantics, the effect system, and the safety theorem in Twelf [13] (Section 8).

\section{Core EFF}

The current implementation of Eff includes a number of features, such as syntactic sugar, products, records, inductive types, type definitions, effect definitions, etc., which are inessential for a conceptual analysis. We therefore restrict attention to core Eff, a fragment of the language described here. We refer the readers to [2] for a more thorough introduction of how one actually programs in Eff.

In Eff all computational effects are accessed uniformly and exclusively through operations. These are a primitive concept, of which typical examples are reading and writing on a communication channel, updating and looking up the contents of a reference, and raising an exception. Thus, in Eff each terminating computation results either in an effect-free value, or it calls an operation. Each operation has an associated delimited continuation, which is a suspended computation awaiting the result of the operation.

Operations do not actually perform effects, but are just suspended computations whose behavior is controlled by a second primitive notion, the effect handlers. These are like exception handlers, except that an effect handler has access to the continuation of the handled operation, and so may restart the computation after the operation is handled. With handlers we may implement all the usual computational effects, as well as great variety of others, such as transactional memory, non-deterministic execution strategies, stream redirection, cooperative multi-threading, and delimited continuations. At the top level there may be built-in handlers that provide interaction with the external environment, although we do not consider these in core Eff.

Since Eff is geared towards practical programming, it and core Eff depart in several respects from previous work on handlers and algebraic effects [19, 7]. First, rather than imposing equations on handlers by a typing discipline, the programmer may write arbitrary handlers, and then prove that a particular handler satisfies the desired equations. We demonstrate the technique in Section 7.1, where we implement a state handler and show that 
it satisfies the standard equations. Second, Eff uses fine-grained call-by-value evaluation strategy [10] rather than the theoretically more desirable call-by-push-value [9] because we found the former to be closer to programming practice as well as easier to implement. Third, every effect has multiple instances. For example, a program may write and read from multiple communication channels, raise different kinds of exceptions, and manipulate multipartite state. Thus in core Eff an operation symbol op is always paired with an instance $\iota$ to give an operation $\iota$ \#op. From a theoretical point of view instances are straightforward (as long as we do not generate them dynamically) and inessential, but are absolutely necessary for practical programming.

2.1. Effects and types. To get things going we presume given a collection of effects Effect $E::=$ exception $\mid$ ref $\mid \cdots$

which in full Eff are declared by the programmer. For each $E$ there is a given set $\mathcal{I}_{E}$ of instances $\iota_{1}, \iota_{2}, \iota_{3}, \ldots$ of $E$. The instances may be thought of as atomic names. In full Eff they may be dynamically created, but to keep the semantics reasonably simple we assume a fixed set. Additionally, with each $E$ we associate a set of $\mathcal{O}_{E}$ of operation symbols $\mathrm{op}_{1}, \mathrm{op}_{2}, \ldots$ An operation symbol is associated with at most one effect.

The terms of core Eff are split into effect-free expressions and possibly effectful computations, as described in the next subsection. Consequently, the type system of core Eff consists of pure types for expressions and dirty types for computations:

$$
\begin{aligned}
& \text { Pure type } A, B::=\text { bool } \mid \text { nat } \mid \text { unit } \mid \text { empty }|A \rightarrow \underline{C}| E^{R} \mid \underline{C} \Rightarrow \underline{D} \\
& \text { Dirty type } \underline{C}, \underline{D}::=A ! \Delta \\
& \text { Region } R::=\left\{\iota_{1}, \ldots, \iota_{n}\right\} \\
& \text { Dirt } \Delta::=\left\{\iota_{1} \# \mathrm{op}_{1}, \ldots, \iota_{n} \# \mathrm{op}_{n}\right\}
\end{aligned}
$$

A dirty type $A ! \Delta$ is just a pure type $A$ tagged with a finite set $\Delta$ of operations that might be called during evaluation. We require that any operation $\iota$ \#p appearing in $\Delta$ is well-formed in the sense that $\iota \in \mathcal{I}_{E}$ and op $\in \mathcal{O}_{E}$ for some effect $E$.

The pure types comprise the usual ground types, the function types $A \rightarrow \underline{C}$, the effect types $E^{R}$, and the handler types $\underline{C} \Rightarrow \underline{D}$. Note that the function type takes pure types to dirty ones because a function accepts a pure expression as an argument and may call operations when evaluated. We let ! bind more strongly than $\rightarrow$, so that $A \rightarrow B$ ! $\Delta$ means $A \rightarrow(B ! \Delta)$. Each effect type $E^{R}$ is tagged with a finite set of instances $R=\left\{\iota_{1}, \ldots, \iota_{n}\right\} \subseteq$ $\mathcal{I}_{E}$ which tells us that the expression equals one of the instances in $R$. Finally, $\underline{C} \Rightarrow \underline{D}$ is the type of handlers which take computations of ingoing type $\underline{C}$ to computations of outgoing type $\underline{D}$.

We assume that each effect $E$ has an associated effect signature

$$
\Sigma_{E}=\left\{\mathrm{op}_{1}: A^{\mathrm{op}_{1}} \rightarrow B^{\mathrm{op}_{1}}, \ldots, \mathrm{op}_{n}: A^{\mathrm{op}_{n}} \rightarrow B^{\mathrm{op}_{n}}\right\}
$$

which assigns to each operation $\mathrm{op}_{i} \in \mathcal{O}_{E}$ its parameter type $A^{\mathrm{op}_{i}}$ and result type $B^{\mathrm{op}_{i}}$. In full Eff the signature is part of the definition of an effect, so for instance we might have

$$
\begin{aligned}
\Sigma_{\text {ref }} & =\{\text { lookup }: \text { unit } \rightarrow \text { nat }, \text { update }: \text { nat } \rightarrow \text { unit }\}, \\
\Sigma_{\text {exception }} & =\{\text { raise }: \text { unit } \rightarrow \text { empty }\}
\end{aligned}
$$


Note that the signature may create circularities such as

$$
\Sigma_{E}=\{\text { op }: \text { unit } \rightarrow(\text { unit } \rightarrow \text { unit } !\{\iota \# \text { op }\})\} .
$$

Consequently, the denotational semantics of types in Section 5 will involve recursive domain equations.

2.2. Terms. The abstract syntax of terms of core Eff is as follows:

Expression $e::=x \mid$ true $\mid$ false $|0|$ succ $e|()|$ fun $x: A \mapsto c|\iota| h$

Handler $h::=$ (handler val $x: A \mapsto c_{v} \mid$ ocs)

Operation cases ocs $::=n i \underline{l}_{\underline{C}} \mid(e \#$ op $x k \mapsto c \mid$ ocs $)$

Computation $c::=\operatorname{val} e \mid e_{1} \#$ op $e_{2}(y . c) \mid$ with $e$ handle $c \mid$

if $e$ then $c_{1}$ else $c_{2}\left|\operatorname{absurd}_{\underline{C}} e\right| e_{1} e_{2} \mid$

(match $e$ with $\left.0 \mapsto c_{1} \mid \operatorname{succ} x \mapsto c_{2}\right) \mid$

let $x=c_{1}$ in $c_{2} \mid$ let rec $f x: A \rightarrow \underline{C}=c_{1}$ in $c_{2}$

In order to ensure that each term has at most one skeletal typing derivation, cf. Subsection 4.3, certain terms include typing annotations. We shall omit these when they do not play a role. The Eff implementation does not have typing annotations because its effect system automatically infers types and effects [22].

An expression is either a variable, a constant of ground type, a function abstraction (note that we abstract over computations), an effect instance, or a handler. It is worth noting that both instances and handlers are first-class values. We sometimes abbreviate $\operatorname{succ}^{n} 0$ as $n$. A handler consists of a single value case and multiple operation cases, which describe how values and operations are handled, respectively. We defined operation cases inductively as lists, which is how they are formalized in Twelf, but we also write them as $\left(e_{i} \# \mathrm{op}_{i} x_{i} k_{i} \mapsto c_{i}\right)_{i}$.

A computation is either a pure expression, an operation call, a handle construct, an eliminator for a ground type, an application, a let binding, or a recursive function definition.

\section{Operational SEMANTiCS}

We first describe the operational semantics informally. A computation val $e$ is pure and indicates a "final" result $e$, while an operation call $e_{1} \# \circ e_{2}(y . c)$ is the principal way of triggering an effect. The instance $e_{1}$ and the operation symbol op together form an operation $e_{1} \#$ op, its parameter is $e_{2}$, and $(y . c)$ the delimited continuation. We do not expect programmers to write explicit continuations, so the concrete syntax of Eff only gives access to calls through functions of the form fun $x \mapsto e \#$ op $x$ ( $y$. val $y$ ), known also as generic effects [17]. In examples we shall use generic effects rather than explicit continuations, and there we write them as e\#op. A general operation call $e_{1} \#$ op $e_{2}(y . c)$ may then be expressed in terms of a generic effect and a let binding as let $y=e_{1} \#$ op $e_{2}$ in $c$.

A binding let $x=c_{1}$ in $c_{2}$ is evaluated as follows:

(1) If $c_{1}$ evaluates to val $e$ then the binding evaluates as $c_{2}$ with $x$ bound to $e$. 
(2) If $c_{1}$ evaluates to an operation call $\iota$ \#op $e\left(y . c_{1}^{\prime}\right)$, then the binding evaluates to

$$
\iota \text { \#op } e\left(y \text {. let } x=c_{1}^{\prime} \text { in } c_{2}\right) \text {, }
$$

where we assume that $y$ does not occur free in $c_{2}$.

It may be helpful to think of val and let as being similar to Haskell return and do, respectively. In ML val is invisible, while let is essentially the same as ours.

The handle construct applies a handler to a computation. If $h$ is the handler

$$
\text { handler val } x \mapsto c_{v} \mathrm{I}\left(\iota_{i} \# \mathrm{op}_{i} x_{i} k_{i} \mapsto c_{i}\right)_{i}
$$

and $c$ is a computation, then with $h$ handle $c$ first evaluates $c$ which is then handled according to $h$ :

(1) If $c$ evaluates to val $e$, then the handle construct evaluates as $c_{v}$ with $x$ bound to $e$.

(2) If $c$ evaluates to $\iota \# \circ \mathrm{pp} e^{\prime}\left(y \cdot c^{\prime}\right)$, and $\iota_{i} \# \circ \mathrm{p}_{i} x_{i} k_{i} \mapsto c_{i}$ is the first operation case in $h$ for which $\iota$ op $=\iota_{i} \# \mathrm{op}_{i}$ then the handle construct evaluates to $c_{i}$ with $x_{i}$ and $k_{i}$ bound to $e^{\prime}$ and fun $y \mapsto$ with $h$ handle $c^{\prime}$, respectively. We assume that $y$ does not occur free in $h$.

(3) If $c$ evaluates to an operation call $\iota$ op $e^{\prime}\left(y \cdot c^{\prime}\right)$ which is not listed by $h$, then the handle construct propagates the call and acts as if $h$ contained the clause

$$
\iota \# \text { op } x k \mapsto \iota \# \text { op } x(y . k y) .
$$

Thus it evaluates to $\iota$ \# $e^{\prime}\left(y\right.$. with $h$ handle $c^{\prime}$ ), where again we assume that $y$ does not occur free in $h$.

Note that the handler always wraps itself around the continuation so that subsequent operations are handled as well. A binding let $x=c_{1}$ in $c_{2}$ is equivalent to

$$
\text { with (handler val } x \mapsto c_{2} \text { ) handle } c_{1}
$$

so we could theoretically omit let.

3.1. Small-step semantics. The small-step operational semantics of core Eff is defined in terms of a relation $c \rightsquigarrow c^{\prime}$, which intuitively means that the computation $c$ takes a single step to $c^{\prime}$. There is no operational semantics for expressions, which are just inert pieces of 
data. The relation $\rightsquigarrow$ is defined inductively by the following rules:

$$
\begin{aligned}
& \overline{\text { if true then } c_{1} \text { else } c_{2} \rightsquigarrow c_{1}} \quad \overline{\text { if false then } c_{1} \text { else } c_{2} \rightsquigarrow c_{2}} \\
& \overline{\left(\text { match } 0 \text { with } 0 \mapsto c_{1} \mid \operatorname{succ} x \mapsto c_{2}\right) \rightsquigarrow c_{1}} \overline{\left(\operatorname{match}(\operatorname{succ} e) \text { with } 0 \mapsto c_{1} \mid \operatorname{succ} x \mapsto c_{2}\right) \rightsquigarrow c_{2}[e / x]} \\
& \frac{c_{1} \rightsquigarrow c_{1}^{\prime}}{(\text { fun } x \mapsto c) e \rightsquigarrow c[e / x]} \quad \frac{\operatorname{let} x=c_{1} \text { in } c_{2} \rightsquigarrow \operatorname{let} x=c_{1}^{\prime} \text { in } c_{2}}{\text { let }} \\
& \overline{\text { let } x=(\operatorname{val} e) \text { in } c \rightsquigarrow c[e / x]} \quad \overline{\text { let } x=\left(\iota \# \text { op } e\left(y . c_{1}\right)\right) \text { in } c_{2} \rightsquigarrow \iota \text { \#op } e\left(y \text {. let } x=c_{1} \text { in } c_{2}\right)} \\
& \overline{\text { let } \operatorname{rec} f x=c_{1} \text { in } c_{2} \rightsquigarrow c_{2}\left[\left(\text { fun } x \mapsto \operatorname{let} \operatorname{rec} f x=c_{1} \text { in } c_{1}\right) / f\right]} \\
& \frac{c \rightsquigarrow c^{\prime}}{\text { with } e \text { handle } c \rightsquigarrow \text { with } e \text { handle } c^{\prime}} \quad \frac{\text { with (handler val } x \mapsto c_{v} \mid \text { ocs) handle }(\operatorname{val} e) \rightsquigarrow c_{v}[e / x]}{x} \\
& \frac{h \stackrel{\text { def }}{=}\left(\text { handler val } x \mapsto c_{v} \mid \text { ocs }\right) \quad\left(\text { op }: A^{\mathrm{op}} \rightarrow B^{\mathrm{op}}\right) \in \Sigma_{E}}{\text { with } h \text { handle }(\iota \# \text { op } e(y . c)) \rightsquigarrow o c s_{\iota \# \text { op }}\left(e,\left(\text { fun } y: B^{\mathrm{op}} \mapsto \text { with } h \text { handle } c\right)\right)}
\end{aligned}
$$

In the last rule for let binding and the last rule for the handle construct variable $y$ must not occur free in $c_{2}$ and $h$ respectively, and it goes without saying that the substitutions are capture avoiding. In the last rule we have an auxiliary definition of ocs $_{\iota \text { \#op }}$ :

$$
\begin{aligned}
(n i l)_{\iota \# \mathrm{op}}(e, \kappa) & =\iota \# \mathrm{op} e(y . \kappa y) \\
\left(\iota^{\prime} \# \mathrm{op}^{\prime} x k \mapsto c \mid \text { ocs }\right)_{\iota \# \mathrm{op}}(e, \kappa) & = \begin{cases}c[e / x, \kappa / k] & \text { if } \iota \# \mathrm{op}=\iota^{\prime} \# \mathrm{op}^{\prime} \\
o c s_{\iota \# o p}(e, \kappa) & \text { otherwise }\end{cases}
\end{aligned}
$$

In words, $o c s_{\iota \# \text { op }}(e, \kappa)$ finds the first handler case in ocs that matches the operation $\iota$ \#op and executes it, or calls the operation again if no match is found.

Example 3.1. The non-standard state handler (recall that in examples we use generic effects)

$$
\begin{aligned}
& h \stackrel{\text { def }}{=} \text { handler } \\
& \mid \text { val } x \mapsto \iota \text { «update } x \\
& \mid \iota \text { lookup } x k \mapsto k 1 \\
& \mid \text { | \#update } x k \mapsto k()
\end{aligned}
$$

treats the reference $\iota$ as if its content were always 1 , and updates $\iota$ with the final result of the handled computation. This update is not handled by $h$ because it escapes its scope. If we use $h$ to handle the computation

$$
\begin{aligned}
c \stackrel{\text { def }}{=} & \text { let } x_{1}=\iota \# \text { lookup }() \text { in } \\
& \text { let } x_{2}=\iota \text { \#update } x_{1} \text { in } \\
& \operatorname{val}\left(\operatorname{succ} x_{1}\right)
\end{aligned}
$$

the outcome of the first lookup is 1 , which is bound to $x_{1}$, the update is ignored and finally ı\#update 2 is called. The exact reduction sequence is as follows, where we underline the 
active parts at each step and indicate desugaring of generic effects with $\equiv$ :

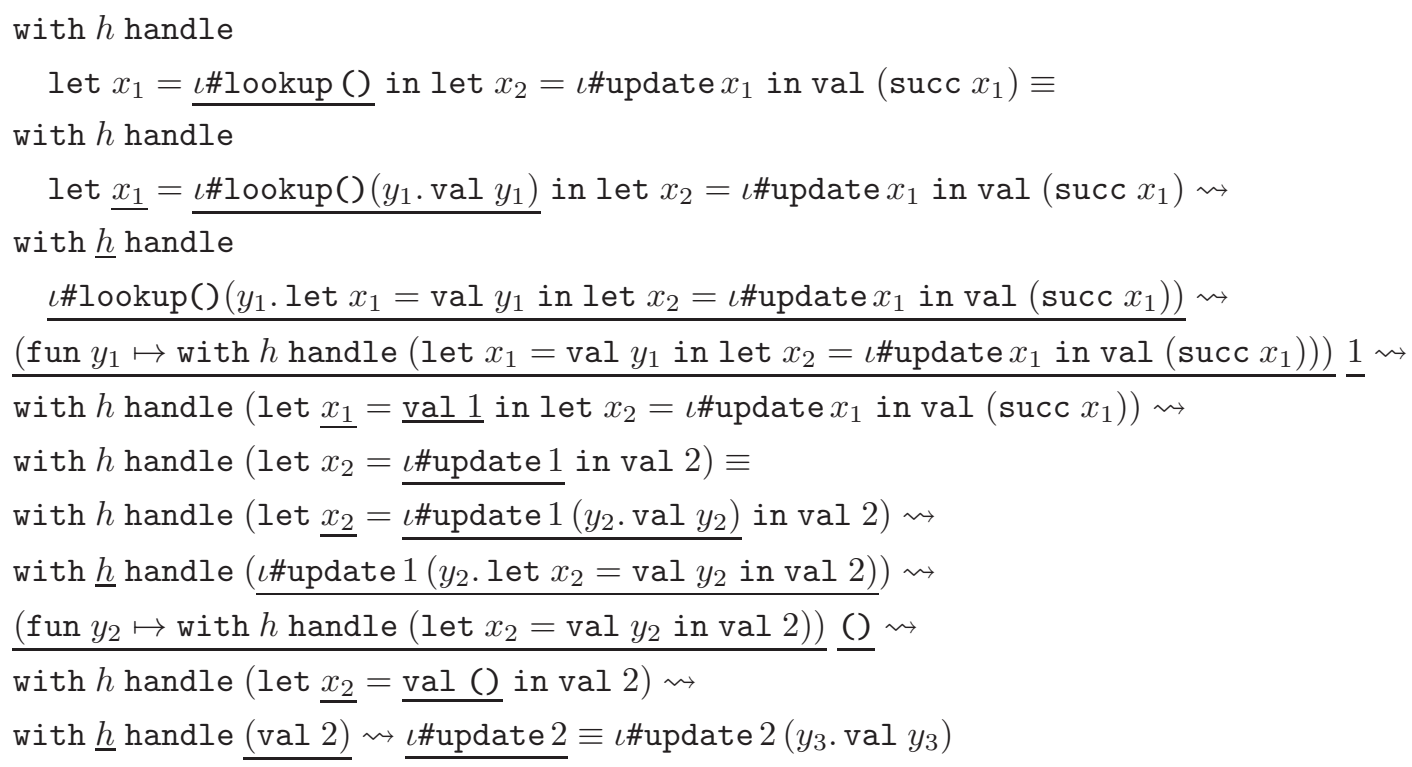

3.2. Big-step semantics. In addition to small-step operational semantics, we also provide a big-step variant, which is closer to the actual implementation of Eff. Define a result to be a pure expression or an operation call:

$$
\text { Result } r::=\operatorname{val} e \mid \iota \text { ॥op } e(x . c)
$$

Big-step semantics $c \Downarrow r$ evaluates a computation $c$ to a result $r$, according to the following inductive rules:

$$
\begin{aligned}
& c_{1} \Downarrow r \\
& \overline{\text { if true then } c_{1} \text { else } c_{2} \Downarrow r} \\
& \frac{c_{2} \Downarrow r}{\text { if false then } c_{1} \text { else } c_{2} \Downarrow r} \\
& \frac{c_{1} \Downarrow r}{\left(\operatorname{match} 0 \text { with } 0 \mapsto c_{1} \mid \operatorname{succ} x \mapsto c_{2}\right) \Downarrow r} \\
& \frac{c_{2}[e / x] \Downarrow r}{\left(\text { match succ } e \text { with } 0 \mapsto c_{1} \mid \operatorname{succ} x \mapsto c_{2}\right) \Downarrow r} \\
& \frac{c[e / x] \Downarrow r}{(\operatorname{fun} x \mapsto c) e \Downarrow r} \quad \frac{c_{1} \Downarrow \operatorname{val} e \quad c_{2}[e / x] \Downarrow r}{\operatorname{val} e \Downarrow \operatorname{val} e} \quad \frac{c_{1}}{\iota \text { et } x=(x . c) \Downarrow \iota \text { in } c_{2} \Downarrow r} \\
& \frac{c_{1} \Downarrow \iota \text { \#op } e(y . c)}{\operatorname{let} x=c_{1} \text { in } c_{2} \Downarrow \iota \text { \#op } e\left(y . \text { let } x=c \text { in } c_{2}\right)} \quad \frac{c_{2}\left[\left(\text { fun } x \mapsto \operatorname{let} \operatorname{rec} f x=c_{1} \text { in } c_{1}\right) / f\right] \Downarrow r}{\operatorname{let} \operatorname{rec} f x=c_{1} \text { in } c_{2} \Downarrow r} \\
& \frac{c \Downarrow \operatorname{val} e \quad c_{v}[e / x] \Downarrow r}{\text { with (handler val } x \mapsto c_{v} \mid \text { ocs) handle } c \Downarrow r} \\
& \left.h \stackrel{\text { def }}{=} \text { (handler val } x \mapsto c_{v} \mid \text { ocs }\right) \quad c \Downarrow \iota \text { ८op } e(y . c) \quad\left(\text { op }: A^{\text {op }} \rightarrow B^{\text {op }}\right) \in \Sigma_{E} \\
& o c s_{\iota \# \text { op }}\left(e,\left(\text { fun } y: B^{\text {op }} \mapsto \text { with } h \text { handle } c\right)\right) \Downarrow r \\
& \text { with } h \text { handle } c \Downarrow r
\end{aligned}
$$


To relate the two semantics we define an auxiliary relation $\rightsquigarrow^{*}$ by the rules

$$
\overline{\operatorname{val} e \rightsquigarrow^{*} \operatorname{val} e} \quad \frac{c \rightsquigarrow c^{\prime} c^{\prime} \rightsquigarrow^{*} r}{c \rightsquigarrow^{*} r}
$$

This is roughly the reflexive transitive closure of $\rightsquigarrow$, except that it relates computations to results rather than to computations. The small-step and big-step semantics agree in the following sense.

Proposition 3.2. For all computations $c$ and results $r, c \Downarrow r$ if and only if $c \rightsquigarrow^{*} r$.

Proof. Both directions of the equivalence proceed by a routine induction. The formalized proofs of the two implications can be found in the file small-big.elf.

\section{An EFFECT SYSTEM}

4.1. Subtyping. As in most effect systems, we need to take care of the poisoning problem [26]. For example, what should be the type of ignore in

$$
\begin{aligned}
& \text { let ignore }=\text { val (fun } m s g \mapsto \text { val }()) \text { in } \\
& \text { let } f=\text { if } b \text { then (val ignore) else (val std\#write) in } \\
& \text { val ignore }
\end{aligned}
$$

assuming we have the ground type string and a boolean expression $b$ ? If we give it the desired type string $\rightarrow$ unit! $\varnothing$ then there is a type mismatch between the branches in the conditional statement, whereas the dirty type string $\rightarrow$ unit! \{std\#write\} loses the valuable knowledge that ignore is a pure function. The simplest antidote to the poisoning problem is subtyping so that ignore may be given the function type with empty dirt which is coerced in the conditional statement to a supertype that matches the other branch.

For our purposes, a straightforward variant of structural subtyping [6] suffices. We have subtyping of pure types $A \leqslant A^{\prime}$ and of dirty types $\underline{C} \leqslant \underline{C}$, given by the rules

$$
\begin{aligned}
& \overline{\text { bool } \leqslant \text { bool }} \quad \overline{\text { nat } \leqslant \text { nat }} \quad \overline{\text { unit } \leqslant \text { unit }} \quad \overline{\text { empty } \leqslant \text { empty }} \quad \frac{A^{\prime} \leqslant A \quad \underline{C} \leqslant \underline{C}^{\prime}}{A \rightarrow \underline{C} \leqslant A^{\prime} \rightarrow \underline{C^{\prime}}} \\
& \frac{R \subseteq R^{\prime}}{E^{R} \leqslant E^{R^{\prime}}} \quad \frac{\underline{C}^{\prime} \leqslant \underline{C} \quad \underline{D} \leqslant \underline{D}^{\prime}}{\underline{C} \Rightarrow \underline{D} \leqslant \underline{C}^{\prime} \Rightarrow \underline{D}^{\prime}} \quad \frac{A \leqslant A^{\prime} \quad \Delta \subseteq \Delta^{\prime}}{A ! \Delta \leqslant A^{\prime} ! \Delta^{\prime}}
\end{aligned}
$$

It is easily checked that reflexivity and transitivity of subtyping are admissible. Apart from resolving the poisoning problem, subtyping allows us to better deduce the behavior of handlers. Consider the computation

$$
\begin{aligned}
& \text { let } u=\operatorname{val} \iota \text { in } \\
& \text { let } v=\left(\text { if } b \text { then val } u \text { else val } \iota^{\prime}\right) \text { in } \\
& \text { let } h=\operatorname{val}(\text { handler val } x \mapsto \cdots \mid u \# \text { op } x k \mapsto c) \text { in }
\end{aligned}
$$

Without subtyping we are forced to give both $u$ and $v$ the type $E^{\left\{\iota, \iota^{\prime}\right\}}$. Therefore, by looking at the type of $u$ we cannot tell whether $h$ handles $\iota$ \# op or $\iota^{\prime} \#$ op, and so we must assume 
that both may be unhandled by $h$. With subtyping we may give $u$ the type $E^{\{\iota\}}$ which makes it clear that $h$ handles ı\#op.

4.2. Typing rules. There are two typing judgments,

$$
\Gamma \vdash e: A \quad \text { and } \quad \Gamma \vdash c: \underline{C}
$$

stating that an expression $e$ has a pure type $A$ and a computation $c$ has a dirty type $\underline{C}$, respectively. Here $\Gamma$ is a typing context of the form $x_{1}: A_{1}, \ldots, x_{n}: A_{n}$. There is also a third, auxiliary typing judgment

$$
\Gamma \vdash \text { ocs }: \underline{C} / \Delta
$$

which states that operation cases ocs all have the same outgoing type $\underline{C}$ and are guaranteed to handle operations in $\Delta$. Most of the typing rules in Figure 1 are standard, except for:

InsT: we check that $\iota$ is one of instances in $R$, which in turn must be contained in $\mathcal{I}_{E}$.

HAND: to check that a handler has type $A ! \Delta \Rightarrow B ! \Delta^{\prime}$, we verify that it converts a computation of type $A ! \Delta$ to one of type $B ! \Delta^{\prime}$, which involves checking three premises. First, the value case must take a value of type $A$ to a computation of type $B ! \Delta^{\prime}$. Second, all operation cases must have outgoing type $B ! \Delta^{\prime}$. Third, every operation in $\Delta$ is either guaranteed to be handled by ocs, or is contained in $\Delta^{\prime}$.

OpCASES-NiL, OpCASEs-Cons: the auxiliary typing judgment verifies that the operation cases have the given outgoing type, and that they cover the given dirt. The empty list nil does not cover anything and has any outgoing type. The rule OpCASEs-Cons checks the first operation case, checks the others inductively, and verifies that $\Delta \subseteq \Delta^{\prime} \cup R \#$ op, where

$$
\Delta^{\prime} \cup R \# \text { op } \stackrel{\text { def }}{=} \begin{cases}\Delta^{\prime} \cup\{\iota \# \text { op }\} & \text { if } R=\{\iota\}, \\ \Delta^{\prime} & \text { otherwise. }\end{cases}
$$

The idea is that we can be sure that an operation case handles ı\#op only when the type of its instance is of the form $E^{\{\iota \# \text { op }\}}$.

Op: we first check that $e$ and op belong to the same effect. Then we check that $\Delta$ covers not just all possible operations that the operation call may cause (recall that $R$ may contain more than one instance), but also any operations in the continuation $c$. We may assume that $c$ has the same dirt, as we can use SubComp otherwise. We use the same reasoning in rules IfThenElse, Match and LET.

Wiтн: handlers behave like functions from computations to computations.

SubExpr, SubComp: these subsumption rules allow us to always assign a bigger type.

The effect system is safe with respect to the operational semantics:

Theorem 4.1 (Progress \& Preservation).

Progress: If $\vdash c: A$ ! $\Delta$ then either

- there exists a computation $c^{\prime}$ such that $c \rightsquigarrow c^{\prime}$, or

- $c$ is of the form val e for some expression e, or

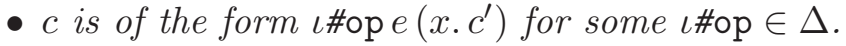

Preservation: If $\vdash c: \underline{C}$ and $c \rightsquigarrow c^{\prime}$ then $\vdash c^{\prime}: \underline{C}$.

Proof. Both statements are proved by induction. The formalized proofs can be found in the files progress.elf and preservation.elf. 


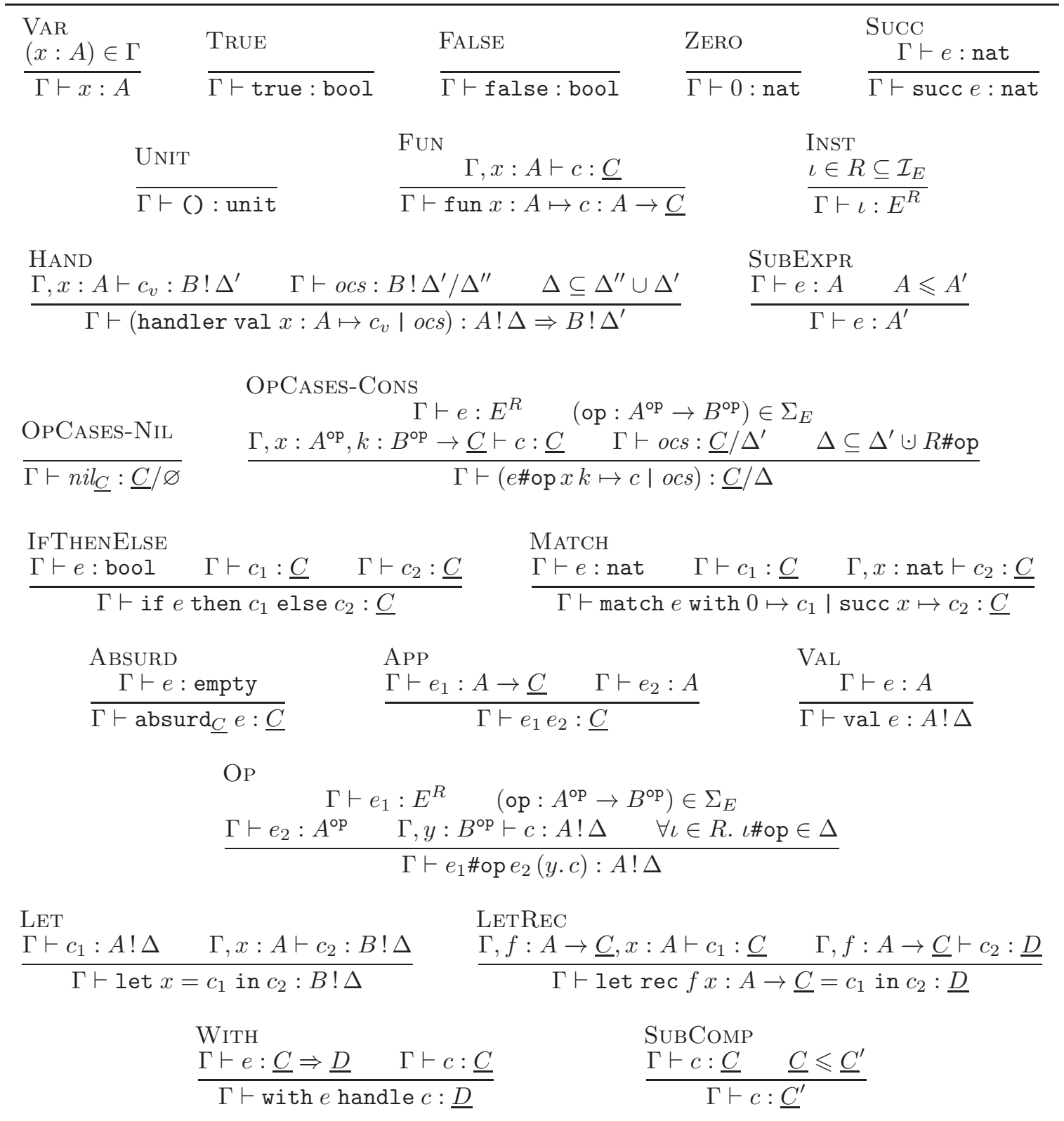

Figure 1: The typing rules of core Eff.

Corollary 4.2 (Safety). A terminating computation of type $A ! \Delta$ returns a value of type $A$, or calls an operation in $\Delta$.

In particular, a terminating computation of type $A ! \varnothing$ does not call any operations and returns a pure value of type $A$.

Example 4.3. Let us see what the effect system tells us about Example 3.1. We may give the reference $\iota \in \mathcal{I}_{\text {ref }}$ type $\operatorname{ref}^{\{\iota\}}$. Then the computation $c$ has the dirty type 
nat! $\{\iota \#$ lookup, ı\#update $\}$, and the handler $h$ the type

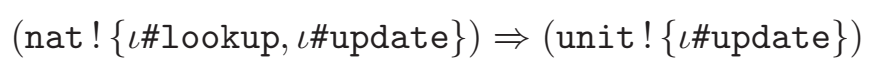

because it handles both lookup and update, but then calls update in the value case. This update also changes the type of computation from nat to unit.

If we give $\iota$ the less precise type $\operatorname{ref}\left\{\iota, \iota^{\prime}\right\}$, the dirt of $c$ is

$$
\Delta \stackrel{\text { def }}{=}\left\{\iota \# \text { lookup, } \iota^{\prime} \# \text { lookup, } \iota \text { \#update }, \iota^{\prime} \text { \#update }\right\}
$$

while the best type we can give to $h$ is (nat $! \Delta) \Rightarrow$ (unit ! $\Delta$ ). Since $\left\{\iota, \iota^{\prime}\right\}$ is not a singleton, we cannot give any guarantees on what operations are handled.

4.3. Skeletal types. We relate the pure and dirty types to ML-style types by an operation which erases all effect information to produce a skeletal type. We will use these later to obtain a coherent semantics of types. The skeletal types are defined as follows:

$$
\text { Skeletal type } S, T::=\text { bool } \mid \text { nat } \mid \text { unit } \mid \text { empty }|S \rightarrow T| E \mid S \Rightarrow T
$$

There is no distinction between pure and dirty types anymore. The typing rules for skeletal types are like those for pure and dirty types with the effect information omitted, for instance

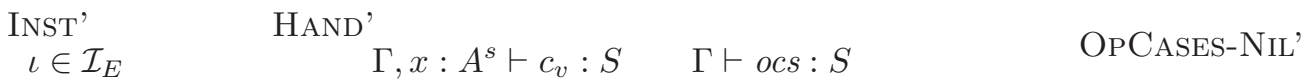

$$
\begin{aligned}
& \frac{\iota \in \mathcal{I}_{E}}{\Gamma \vdash \iota: E} \quad \frac{\Gamma, x: A^{s} \vdash c_{v}: S \quad \Gamma \vdash \text { ocs }: S}{\Gamma \vdash\left(\text { handler val } x: A \mapsto c_{v} \mid \text { ocs }\right): A^{s} \Rightarrow S} \quad \frac{}{\Gamma \vdash n i l_{\underline{C}}: \underline{C}^{s}}
\end{aligned}
$$

OpCASES-CONS'

$\frac{\Gamma \vdash e: E \quad\left(\text { op }: A^{\mathrm{op}} \rightarrow B^{\mathrm{op}}\right) \in \Sigma_{E} \quad \Gamma, x:\left(A^{\mathrm{op}}\right)^{s}, k:\left(B^{\mathrm{op}}\right)^{s} \rightarrow S \vdash c: S \quad \Gamma \vdash \text { ocs }: S}{\Gamma \vdash(e \# \text { op } x k \mapsto c \mid \text { ocs }): S}$

$$
\frac{\begin{array}{l}
\text { OP' } \\
\Gamma \vdash e_{1}: E
\end{array} \quad\left(\text { op }: A^{\mathrm{op}} \rightarrow B^{\mathrm{op}}\right) \in \Sigma_{E} \quad \Gamma \vdash e_{2}:\left(A^{\mathrm{op}}\right)^{s} \quad \Gamma, y:\left(B^{\mathrm{op}}\right)^{s} \vdash c: S}{\Gamma \vdash e_{1} \# \mathrm{op} e_{2}(y \cdot c): S}
$$

The remaining rules remain unchanged as they do not mention effects, while subsumption rules are removed. To every pure type $A$ and a dirty type $\underline{C}$ we assign their skeletal versions $A^{s}$ and $\underline{C}^{s}$, which are like $A$ and $\underline{C}$ with region and dirt removed. The skeletal version $\Gamma^{s}$ of a typing context $\Gamma$ is obtained by taking the skeletons of the types in $\Gamma$. We summarize the properties of skeletal types:

\section{Theorem 4.4.}

(1) If $A \leqslant B$ and $\underline{C} \leqslant \underline{D}$ then $A^{s}=B^{s}$ and $\underline{C}^{s}=\underline{D}^{s}$.

(2) If

then

$$
\Gamma \vdash e: A \quad \text { and } \quad \Gamma \vdash c: \underline{C}
$$

$$
\Gamma^{s} \vdash e: A^{s} \quad \text { and } \quad \Gamma^{s} \vdash c: \underline{C}^{s} .
$$

(3) In a given context, an expression and a computation has at most one skeletal type, with a unique typing derivation. 
Proof. The first statement holds by an induction on the derivation of $A \leqslant B$ and $\underline{C} \leqslant \underline{D}$.

To prove the second statement, observe that a typing derivation may be mapped to the corresponding skeletal version rule by rule, except for subsumption rules. But these can be simply omitted from the typing derivations because $A \leqslant B$ and $\underline{C} \leqslant \underline{D}$ imply $A^{s}=B^{s}$ and $\underline{C}^{s}=\underline{D}^{s}$ by the first statement.

The last statement holds by inversion: in any situation at most one skeletal typing rule applies in at most one way.

A consequence of the theorem is that if a computation $c$ has dirty types $\underline{C}$ and $\underline{D}$ then $\underline{C}^{s}=\underline{D}^{s}$, hence $\underline{C}$ and $\underline{D}$ differ only in the effect information. An analogous property holds for expressions and pure types.

\section{Denotational semantics}

We use standard domain theory to provide an adequate denotational semantics of core Eff. We shall use $\omega$-cpos as domains, but presumably a different kind of domains could be used, as long as they support the standard constructions, in particular solutions of domain equations, and are amenable to Pitts's theory of minimal invariant properties [14]. We refer to [1] for background on domain theory and denotational semantics.

We define a predomain to be a poset in which chains (ascending sequences) have suprema, while a domain is a predomain with a least element $\perp$. A continuous map is a monotone map which commutes with suprema of chains. If $D$ is a predomain and $E$ is a domain the set $D \rightarrow E$ of all continuous maps forms a domain. The ordering on continuous maps is pointwise. A continuous map is strict if it maps $\perp$ to $\perp$. The set $D \multimap E$ of strict maps between domains $D$ and $E$ forms a subdomain of $D \rightarrow E$.

5.1. Computation domains. We first build domains that will serve as the meanings of computation types. Let $A$ be a predomain, $I$ an index set, and for each $i \in I$ let $A_{i}$ and $B_{i}$ be predomains. We seek a domain $T$ satisfying the domain equation $T \cong F(T)$ where $F$ is the functor

$$
F(D)=\left(A+\coprod_{i \in I} A_{i} \times\left(B_{i} \rightarrow D\right)\right)_{\perp} .
$$

Following [14] we work in the category of domains and strict maps, and take $T$ to be a minimal solution in the sense that it possesses the minimal invariant property. The usual limit-colimit construction [1, Chapter 7] yields such a domain. As domain equations go, this one is quite simple because $T$ occurs only covariantly. The elements of $T$ can be thought of as trees whose leaves are tagged with elements of $A$ or $\perp$, and whose nodes have branching types $B_{i}$ and are tagged with elements of $A_{i}$. The trees need not be well founded.

The minimality of $T$ yields a recursion and an induction principle. The recursion principle says that for any domain $D$, a continuous map $f_{\text {val }}: A \rightarrow D$, and continuous maps $f_{i}: A_{i} \times\left(B_{i} \rightarrow D\right) \rightarrow D$ for $i \in I$, there is a unique strict continuous map $f: T \multimap D$ such that

$$
\begin{aligned}
f\left(\operatorname{in}_{\mathrm{val}}(x)\right) & =f_{\mathrm{val}}(x) & & \text { for } x \in A, \\
f\left(\operatorname{in}_{i}(y, \kappa)\right) & =f_{i}(y, f \circ \kappa) & & \text { for } y \in A_{i} \text { and } \kappa: B_{i} \rightarrow T .
\end{aligned}
$$

The induction principle for $T$ applies to admissible predicates on $T$, i.e., those that hold for $\perp$ and are closed under suprema of chains. Precisely, if $\phi$ is an admissible predicate on $T$ such that 
(1) $\phi\left(\operatorname{in}_{\mathrm{val}}(x)\right)$ for all $x \in A$, and

(2) for all $i \in I, x \in A_{i}$, and $\kappa: B_{i} \rightarrow T$, if $\forall y \in B_{i} . \phi(\kappa(y))$ then $\phi\left(\operatorname{in}_{i}(x, \kappa)\right)$,

then $\phi(t)$ holds for all $t \in T$.

For any $I$, the construction of a minimal solution $T_{I}\left(A,\left(A_{i}\right)_{i},\left(B_{i}\right)_{i}\right)$ from the input data $A,\left(A_{i}\right)_{i \in I},\left(B_{i}\right)_{i \in I}$ forms a locally continuous functor

$$
T_{I}: \mathbf{p C p o} \times \mathbf{p C p o}^{I} \times\left(\mathbf{p C} \mathbf{C p o}{ }^{\mathrm{op}}\right)^{I} \rightarrow \mathbf{C p p o} .
$$

Here Cppo is the category of domains and strict continuous maps, while pCpo is the category of predomains and partial continuous maps which are defined on open subsets (an upper set which is inaccessible by suprema of chains). The functor will appear later on in a larger system of recursive domain equations.

5.2. Semantics of skeletal types. We interpret pure and dirty types as predomains and domains, respectively. A typing context is interpreted as a cartesian product of predomains, and a typing judgment as a continuous map. However, typing judgments do not have unique derivations because of the subsumption rules, and so we have to worry about coherence. That is, when we define the meaning of a typing judgment by induction on its derivation, we need to make sure that the result does not depend on the choice of derivation. We accomplish this by providing a semantics which factors through the skeletal types from Section 4.3 ,

Let

$$
\Omega=\left\{\iota \# \text { op } \mid \exists E . \iota \in \mathcal{I}_{E} \wedge \text { op } \in \Sigma_{E}\right\}
$$

be the set of all operations. If dirts could be infinite, $A$ ! $\Omega$ would be a dirty type expressing the fact that any effect could happen.

To each skeletal type $S$ we assign a predomain $\llbracket S \rrbracket_{e}$ and a domain $\llbracket S \rrbracket_{c}$ as follows:

$$
\begin{array}{rlrl}
\llbracket \text { bool } \rrbracket_{e} & =\{\mathrm{ff}, \mathrm{tt}\}, & \llbracket \text { nat } \rrbracket_{e} & =\mathbb{N}, \\
\llbracket \text { unit } \rrbracket_{e} & =\{\star\}, & \llbracket \text { empty } \rrbracket_{e} & =\varnothing, \\
\llbracket S \rightarrow T \rrbracket_{e} & =\llbracket S \rrbracket_{e} \rightarrow \llbracket T \rrbracket_{c}, & \llbracket E \rrbracket_{e} & =\mathcal{I}_{E}, \\
\llbracket S \Rightarrow T \rrbracket_{e} & =\llbracket S \rrbracket_{c} \rightarrow \llbracket T \rrbracket_{c}, &
\end{array}
$$

and

$$
\llbracket S \rrbracket_{c}=T_{\Omega}\left(\llbracket S \rrbracket_{e},\left(\llbracket\left(A^{\mathrm{op}}\right)^{s} \rrbracket_{e}\right)_{\iota \# \mathrm{op}},\left(\llbracket\left(B^{\mathrm{op}}\right)^{s} \rrbracket_{e}\right)_{\iota \# \mathrm{op}}\right) .
$$

These should be read as a system of domain and predomain equations indexed by the skeletal types. There are possible circularities in the system because the equation for $\llbracket S \rrbracket_{c}$ refers to possibly larger skeletal types $\left(A^{\mathrm{op}}\right)^{s}$ and $\left(B^{\mathrm{op}}\right)^{s}$. As in the case of computation domains, we take the minimal solutions which enjoy the minimal invariant property.

To each pure type $A$ and dirty type $\underline{C}$ we assign a skeletal predomain $\llbracket A \rrbracket$ and skeletal domain $\llbracket \underline{C} \rrbracket$ by setting

$$
\llbracket A \rrbracket=\llbracket A^{s} \rrbracket_{e} \quad \text { and } \quad \llbracket \underline{C} \rrbracket=\llbracket \underline{C}^{s} \rrbracket_{c} .
$$

The first part of Theorem 4.4 guarantees that $A \leqslant B$ and $\underline{C} \leqslant \underline{D}$ imply $\llbracket A \rrbracket=\llbracket B \rrbracket$ and $\llbracket \underline{C} \rrbracket=\llbracket \underline{D} \rrbracket$. 
5.3. Semantics of expressions and computations. The meaning of a typing context $\Gamma$

$$
x_{1}: A_{1}, \ldots, x_{n}: A_{n}
$$

is

$$
\llbracket \Gamma \rrbracket=\llbracket A_{1} \rrbracket \times \cdots \times \llbracket A_{n} \rrbracket .
$$

We interpret typing judgments

$$
\Gamma \vdash e: A \quad \text { and } \quad \Gamma \vdash c: \underline{C}
$$

as continuous maps

$$
\llbracket \Gamma \vdash e: A \rrbracket: \llbracket \Gamma \rrbracket \rightarrow \llbracket A \rrbracket \quad \text { and } \quad \llbracket \Gamma \vdash c: \underline{C} \rrbracket: \llbracket \Gamma \rrbracket \rightarrow \llbracket \underline{C} \rrbracket .
$$

When no confusion can arise we abbreviate these as $\llbracket e \rrbracket$ and $\llbracket c \rrbracket$. The definition proceeds by induction on the derivation of the typing judgment. Given an environment $\eta \in \llbracket \Gamma \rrbracket$, the base rules for expressions and the successor rule are taken care of by

$$
\begin{aligned}
& \llbracket \Gamma \vdash x_{i}: A_{i} \rrbracket \eta=\eta_{i} \quad \llbracket \Gamma \vdash \text { false }: \text { bool } \rrbracket \eta=\mathrm{ff} \\
& \llbracket \Gamma \vdash(): \text { unit } \rrbracket=\star \quad \llbracket \Gamma \vdash \text { true : bool } \eta \eta=t \\
& \llbracket \Gamma \vdash 0: \operatorname{nat} \rrbracket \eta=0 \quad \llbracket \Gamma \vdash \operatorname{succ} e: \operatorname{nat} \rrbracket \eta=(\llbracket \Gamma \vdash e: \text { nat } \rrbracket \eta)+1 \\
& \llbracket \Gamma \vdash \iota: E^{R} \rrbracket \eta=\iota,
\end{aligned}
$$

and the abstraction rule by

$$
\llbracket \Gamma \vdash(\text { fun } x: A \mapsto c): A \rightarrow \underline{C} \rrbracket \eta=\lambda a \in \llbracket A \rrbracket \cdot \llbracket \Gamma, x: A \vdash c: \underline{C} \rrbracket(\eta, a)
$$

For the handler rule we set

$$
\llbracket \Gamma \vdash\left(\text { handler val } x: A \mapsto c_{v} \mid \text { ocs }\right): A ! \Delta \Rightarrow B ! \Delta^{\prime} \rrbracket=h
$$

where $h: \llbracket \Gamma \rrbracket \rightarrow \llbracket A ! \Delta \rrbracket \multimap \llbracket B ! \Delta^{\prime} \rrbracket$ is defined by recursion on $\llbracket A ! \Delta \rrbracket$ :

$$
\begin{aligned}
h(\eta)(\perp) & =\perp \\
h(\eta)\left(\operatorname{in}_{\mathrm{val}}(a)\right) & =\llbracket \Gamma, x: A \vdash c_{v}: B ! \Delta^{\prime} \rrbracket(\eta, a) \\
h(\eta)\left(\operatorname{in}_{\iota \# \mathrm{op}}(a, \kappa)\right) & =\llbracket o c s \rrbracket_{\iota \# \mathrm{op}}(\eta, a, h(\eta) \circ \kappa)
\end{aligned}
$$

The auxiliary map

$$
\llbracket o c s \rrbracket_{\iota \# \mathrm{op}}: \llbracket \Gamma \rrbracket \times \llbracket A^{\mathrm{op}} \rrbracket \times \llbracket B^{\mathrm{op}} \rightarrow B ! \Delta^{\prime} \rrbracket \rightarrow \llbracket B ! \Delta^{\prime} \rrbracket
$$

is defined by

$$
\begin{aligned}
& \llbracket n i l_{\underline{C}} \rrbracket_{\iota \# \mathrm{op}}(\eta, a, \kappa)=\operatorname{in}_{\iota \# \mathrm{op}}(a, \kappa) \\
& \llbracket e^{\prime} \# \mathrm{op}^{\prime} x k \mapsto c \mid \text { ocs } \rrbracket_{\iota \# \mathrm{op}}(\eta, a, \kappa)= \\
& \begin{cases}\llbracket \Gamma, x: A^{\mathrm{op}}, k: B^{\mathrm{op}} \rightarrow B ! \Delta^{\prime} \vdash c: B ! \Delta^{\prime} \rrbracket(\eta, a, \kappa) & \text { if }\left(\llbracket e^{\prime} \rrbracket \eta\right) \# \mathrm{op}^{\prime}=\iota \# \mathrm{op}, \\
\llbracket o c s \rrbracket_{\iota \# \mathrm{op}}(\eta, a, \kappa) & \text { otherwise. }\end{cases}
\end{aligned}
$$

Finally, if $\Gamma \vdash e: A^{\prime}$ is derived from the premises $\Gamma \vdash e: A$ and $A \leqslant A^{\prime}$ by the subsumption rule, we set

$$
\llbracket \Gamma \vdash e: A^{\prime} \rrbracket \eta=\llbracket \Gamma \vdash e: A \rrbracket \eta
$$


The definition is meaningful because $A \leqslant A^{\prime}$ implies $\llbracket A \rrbracket=\llbracket A^{\prime} \rrbracket$. The meaning of pure computations and operations is

$$
\begin{aligned}
\llbracket \Gamma \vdash \operatorname{val} e: A ! \Delta \rrbracket \eta & =\mathrm{in}_{\text {val }}(\llbracket \Gamma \vdash e: A \rrbracket) \\
\llbracket \Gamma \vdash e_{1} \# \text { op } e_{2}(y \cdot c): A ! \Delta \rrbracket \eta & =\operatorname{in}_{\llbracket e_{1} \rrbracket \eta \text { op }}\left(\llbracket e_{2} \rrbracket \eta, \lambda b \in \llbracket B^{\mathrm{op}} \rrbracket \cdot \llbracket \Gamma, y: B^{\mathrm{op}} \vdash c: A ! \Delta \rrbracket(\eta, b)\right) .
\end{aligned}
$$

The meaning of elimination forms is

$$
\begin{aligned}
\llbracket \Gamma \vdash \text { with } e \text { handle } c: \underline{D} \rrbracket \eta= & (\llbracket \Gamma \vdash e: \underline{C} \Rightarrow \underline{D} \rrbracket \eta)(\llbracket \Gamma \vdash c: \underline{C} \rrbracket \eta) \\
\llbracket \Gamma \vdash \text { if } e \text { then } c_{1} \text { else } c_{2}: \underline{C} \rrbracket \eta & = \begin{cases}\llbracket \Gamma \vdash c_{1}: \underline{C} \rrbracket \eta & \text { if } \llbracket \Gamma \vdash e: \text { bool } \rrbracket \eta=\mathrm{tt}, \\
\llbracket \Gamma \vdash c_{2}: \underline{C} \rrbracket \eta & \text { if } \llbracket \Gamma \vdash e: \text { bool } \rrbracket \eta=\mathrm{ff}\end{cases} \\
\llbracket \Gamma \vdash \text { absurd } e: \underline{C} \rrbracket \eta & =\perp \\
\llbracket \Gamma \vdash e_{1} e_{2}: \underline{C} \rrbracket \eta & =\left(\llbracket \Gamma \vdash e_{1}: A \rightarrow \underline{C} \rrbracket \eta\right)\left(\llbracket \Gamma \vdash e_{2}: A \rrbracket\right)
\end{aligned}
$$

and

$$
\begin{aligned}
& \llbracket \Gamma \vdash\left(\operatorname{match} e \text { with } 0 \mapsto c_{1} \mid \operatorname{succ} x \mapsto c_{2}\right): \underline{C} \rrbracket \eta= \\
& \begin{cases}\llbracket \Gamma \vdash c_{1}: \underline{C} \rrbracket \eta & \text { if } \llbracket \Gamma \vdash e: \text { nat } \rrbracket \eta=0, \\
\llbracket \Gamma, x: \text { nat } \vdash c_{2}: \underline{C} \rrbracket(\eta, n) & \text { if } \llbracket \Gamma \vdash e: \text { nat } \rrbracket \eta=n+1 .\end{cases}
\end{aligned}
$$

To give semantics of let binding, we first define the lifting of a map $f: \llbracket A \rrbracket \rightarrow \llbracket B ! \Delta \rrbracket$ to be the map $f^{\dagger}: \llbracket A ! \Delta \rrbracket \rightarrow \llbracket B ! \Delta \rrbracket$ defined recursively by

$$
\begin{aligned}
f^{\dagger}(\perp) & =\perp, \\
f^{\dagger}\left(\operatorname{in}_{\mathrm{val}}(x)\right) & =f(x), \\
f^{\dagger}\left(\operatorname{in}_{\iota \# \mathrm{op}}(x, \kappa)\right) & =\operatorname{in}_{\iota \# \mathrm{op}}\left(x, f^{\dagger} \circ \kappa\right) .
\end{aligned}
$$

Then we set

$$
\begin{aligned}
& \llbracket \Gamma \vdash \operatorname{let} x=c_{1} \text { in } c_{2}: B ! \Delta \rrbracket \eta= \\
&\left(\lambda a \in \llbracket A \rrbracket \cdot \llbracket \Gamma, x: A \vdash c_{2}: B ! \Delta \rrbracket(\eta, a)\right)^{\dagger}\left(\llbracket \Gamma \vdash c_{1}: A ! \Delta \rrbracket \eta\right) .
\end{aligned}
$$

The meaning of a recursive function definition is

$$
\llbracket \Gamma \vdash\left(\text { let } \operatorname{rec} f x: A \rightarrow \underline{C}=c_{1} \text { in } c_{2}\right): \underline{C} \rrbracket \eta=\llbracket \Gamma, f: A \rightarrow \underline{C} \vdash c_{2}: \underline{C} \rrbracket(\eta, g)
$$

where $g: \llbracket A \rrbracket \rightarrow \llbracket \underline{C} \rrbracket$ is the least fixed-point of the map

$$
g \mapsto\left(\lambda a \in \llbracket A \rrbracket \cdot \llbracket c_{1} \rrbracket(\eta, g, a)\right) .
$$

Finally, just like for expressions, if $\Gamma \vdash c: \underline{C^{\prime}}$ is derived from the premises $\Gamma \vdash c: \underline{C}$ and $\underline{C} \leqslant \underline{C^{\prime}}$ by the subsumption rule, we set

$$
\llbracket \Gamma \vdash c: \underline{C^{\prime}} \rrbracket \eta=\llbracket \Gamma \vdash c: \underline{C} \rrbracket \eta .
$$

This concludes the definition of denotational semantics of expressions and computations.

Theorem 5.1 (Coherence). All derivations of a typing judgment give it the same meaning.

Proof. The semantics of $\Gamma \vdash e: A$ and $\Gamma \vdash c: \underline{C}$ factor through the associated skeletal derivations $\Gamma^{s} \vdash e: A^{s}$ and $\Gamma^{s} \vdash c: \underline{C}^{s}$, respectively. This is so because the semantic rules given above clearly factor through the associated skeletal rules. In other words, they ignore the effect information and the subsumption rules. Uniqueness of meaning is thus a consequence of the uniqueness of skeletal derivations, cf. Theorem 4.4. 
5.4. Semantics of effects. In the terminology of John Reynolds [23] the semantics given so far is intrinsic, while the semantics of effects given below is extrinsic. This is in accordance with our understanding that effect information is descriptive rather than prescriptive: a function $f$ of type $(A \rightarrow B ! \Delta) \rightarrow B^{\prime} ! \Delta^{\prime}$ should accept any function $g$ of type $A \rightarrow B ! \Delta^{\prime \prime}$, even if $\Delta^{\prime \prime} \nsubseteq \Delta$, although it has the property that if $\Delta^{\prime \prime} \subseteq \Delta$ then $f(g)$ calls only operations in $\Delta^{\prime}$.

For each pure type $A$ and dirty type $\underline{C}$ we define subpredomains $\llbracket A \rrbracket \subseteq \llbracket A \rrbracket$ and subdomains $\llbracket \underline{C} \rrbracket \subseteq \llbracket \underline{C} \rrbracket$ of those elements that behave according to the effect information. The ground types are easy:

$$
\begin{aligned}
\llbracket \text { bool } \rrbracket & =\llbracket \text { bool } \rrbracket, & \llbracket \text { nat } \rrbracket & =\llbracket \text { nat } \rrbracket, \\
\llbracket \text { empty } \rrbracket & =\llbracket \text { empty } \rrbracket, & \llbracket E^{R} \rrbracket & =R .
\end{aligned}
$$

For function types, handler types, and dirty types we would like to solve the following system of equations with unknowns $\llbracket A \rrbracket$ and $\llbracket \underline{C} \rrbracket$ where $A$ and $\underline{C}$ range over pure and dirty types, respectively:

$$
\begin{aligned}
& \llbracket A \rightarrow \underline{C} \rrbracket=\{f \in \llbracket A \rrbracket \rightarrow \llbracket \underline{C} \rrbracket \mid \forall x \in \llbracket A \rrbracket . f(x) \in \llbracket \underline{C} \rrbracket\}, \\
& \llbracket \underline{C} \Rightarrow \underline{D} \rrbracket=\{h \in \llbracket \underline{C} \rrbracket \multimap \llbracket \underline{D} \rrbracket \mid \forall t \in \llbracket \underline{C} \rrbracket . h(t) \in \llbracket \underline{D} \rrbracket\}, \\
& \llbracket A ! \Delta \rrbracket=\left\{t \in \llbracket A ! \Delta \rrbracket \mid t=\perp \vee\left(\exists x \in \llbracket A \rrbracket . t=\operatorname{in}_{\mathrm{val}}(x)\right) \vee\right. \\
& \exists \iota \# \mathrm{op} \in \Delta, y \in \llbracket A^{\mathrm{op}} \rrbracket, \kappa \in \llbracket B^{\mathrm{op}} \rightarrow A ! \Delta \rrbracket . \\
& \left.\left(\forall z \in \llbracket B^{\mathrm{op}} \rrbracket . \kappa(b) \in \llbracket A ! \Delta \rrbracket\right) \wedge t=\operatorname{in}_{\iota \# \mathrm{op}}(y, \kappa)\right\} .
\end{aligned}
$$

The last rule says that $t \in \llbracket A ! \Delta \rrbracket$ when it is $\perp$, or of the form $\operatorname{in}_{\text {val }}(x)$ for some $x \in \llbracket A \rrbracket$, or of the form $\operatorname{in}_{\iota \# \mathrm{op}}(y, \kappa)$ for some $y \in \llbracket A^{\mathrm{op}} \rrbracket$ and $\kappa \in \llbracket B^{\mathrm{op}} \rightarrow A ! \Delta \rrbracket$.

The system is potentially problematic because the types $A^{\mathrm{op}}$ and $B^{\mathrm{op}}$ introduce circularities in the last equation. We apply Pitts's theorem [14, Theorem 4.16] about existence of invariant relations to obtain a solution that satisfies an induction principle, see Theorem 5.2 below. For Pitts's theorem to apply we must verify that our conditions form an admissible action on admissible relations, as defined in [14, Definition 4.6]. The relational structure in question is that of [14, Example 4.2(ii)] for which the accompanying notion of admissibility is the one we are using, cf. [14, Example 4.5(ii)]. The locally continuous functor is the evident one, while its admissible action on relations is read off (5.1). The admissibility of the action follows from [14, Lemma 6.6]: the first two actions in (5.1) are considered explicitly, while the third one is a composition of actions from the cited lemma. The solution so obtained possesses the following induction principle.

Theorem 5.2. Suppose $\phi$ is an admissible predicate on $\llbracket A ! \Delta \rrbracket$ such that

(1) $\phi\left(\operatorname{in}_{\mathrm{val}}(a)\right)$ for every $a \in \llbracket A \rrbracket$, and

(2) for all ı\#op $\in \Delta, a \in \llbracket A^{\mathrm{op}} \rrbracket, \kappa \in \llbracket B^{\mathrm{op}} \rightarrow A ! \Delta \rrbracket$, if $\forall b \in \llbracket B^{\mathrm{op}} \rrbracket . \phi(\kappa(b))$ then $\phi\left(\operatorname{in}_{\iota \# \mathrm{op}}(a, \kappa)\right)$.

Then $\phi(t)$ for all $t \in \llbracket A ! \Delta \rrbracket$.

Proof. Because we defined $\mathbb{I - \rrbracket}$ mutually for all types, we first extend $\phi$ to be constantly true on types other than $A ! \Delta$. Then our theorem becomes an instance of the induction property [14, Theorem 6.5]. The admissible action required for the application of the theorem is obtained using [14, Lemma 6.6]. 
The semantics of effects and the semantics of expressions and computations fit together:

Theorem 5.3. If $\Gamma \vdash e: A$ and $\Gamma \vdash c: \underline{C}$ then for all $\eta \in \llbracket \Gamma \rrbracket=\llbracket A_{1} \rrbracket \times \cdots \times \llbracket A_{n} \rrbracket$, we have

$$
\llbracket \Gamma \vdash e: A \rrbracket \eta \in \llbracket A \rrbracket \quad \text { and } \quad \llbracket \Gamma \vdash c: \underline{C} \rrbracket \eta \in \llbracket C \rrbracket .
$$

Proof. The proof proceeds by induction on the derivation of the typing judgment. All cases are easy, except for the typing rule for a handler

$$
\Gamma \vdash\left(\text { handler val } x \mapsto c_{v} \mid \text { ocs }\right): A ! \Delta \Rightarrow B ! \Delta^{\prime}
$$

First, we claim that if the auxiliary judgment

$$
\Gamma \vdash \text { ocs }: B ! \Delta^{\prime} / \Delta_{c}
$$

is derivable then $\llbracket o c s \rrbracket_{\iota \# \mathrm{op}}(\eta, a, \kappa) \in \llbracket B ! \Delta^{\prime} \rrbracket$ for all $\iota$ op $\in \Delta^{\prime} \cup \Delta_{c}, \eta \in \llbracket \Gamma \rrbracket, a \in \llbracket A^{\mathrm{op}} \rrbracket$, and $\kappa: \llbracket B^{\text {op }} \rightarrow B ! \Delta^{\prime} \rrbracket$. Assuming the claim has been established, the above handler rule follows immediately.

The proof of the claim proceeds by induction on the derivation of the auxiliary judgment. For nil, we have $\Delta_{c}=\varnothing$ and the claim obviously holds. The other possibility is

$$
\Gamma \vdash(\text { e\#op' } x k \mapsto c \mid \text { ocs }): B ! \Delta^{\prime} / \Delta_{c}^{\prime}
$$

for some $\Gamma \vdash$ ocs $: B ! \Delta^{\prime} / \Delta_{c}$ and $\Delta_{c}^{\prime} \subseteq\left(\Delta_{c} \bullet R \#\right.$ op $)$. Define $\iota^{\prime}=\llbracket \Gamma \vdash e: E^{R} \rrbracket \eta$ and consider two cases. First, if $\iota^{\prime} \# \mathrm{op}^{\prime}=\iota$ \#op then

$$
\llbracket e \# \mathrm{op}^{\prime} x k \mapsto c \mid \text { ocs } \rrbracket_{\iota \# \mathrm{op}}(\eta, a, \kappa)=\llbracket \Gamma, x: A^{\mathrm{op}}, k: B^{\mathrm{op}} \rightarrow B ! \Delta^{\prime} \vdash c: B ! \Delta^{\prime} \rrbracket(\eta, a, \kappa),
$$

and we may apply the induction hypothesis (for the whole theorem) to $c$. Second, suppose

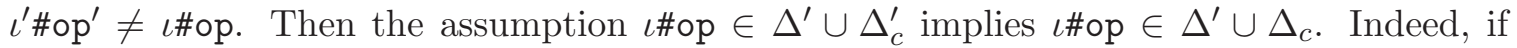
$\iota$ ॥ op $\in \Delta^{\prime}$ there is nothing to prove, and if $\iota$ op $\in \Delta_{c}^{\prime} \subseteq\left(\Delta_{c} \cup R \#\right.$ op), then either $R$ is not a singleton and $\Delta_{c} \cup R \# \mathrm{op}=\Delta_{c}$, or $R=\left\{\iota^{\prime} \# \mathrm{op}^{\prime}\right\}$ and $\iota \#$ op $\notin R$. In any case, it follows that

$$
\llbracket e \# \mathrm{op}^{\prime} x k \mapsto c \mid \text { ocs } \rrbracket_{\iota \# \mathrm{op}}(\eta, a, \kappa)=\llbracket o c s \rrbracket_{\iota \# \mathrm{op}}(\eta, a, \kappa)
$$

and $\Gamma \vdash$ ocs $: B ! \Delta^{\prime} / \Delta_{c}$. Thus we may apply the induction hypothesis.

5.5. Soundness and adequacy. The soundness and adequacy theorems state that operational semantics and denotational semantics fit together. One is an easy induction, while the other is proved using standard technique of formal approximation relations, e.g., see [1, Theorem 6.3.6].

Theorem 5.4 (Soundness). If $\vdash c: \underline{C}$ and $c \rightsquigarrow c^{\prime}$ then $\llbracket \vdash c: \underline{C} \rrbracket=\llbracket \vdash c^{\prime}: \underline{C} \rrbracket$.

Proof. We just have to walk through all the defining rules for $\rightsquigarrow$ and verify that they do indeed preserve the meaning of $c$. 
To tackle adequacy, see Corollary 5.8 below, we define formal approximation relations $\triangleleft_{A}$ and $\triangleleft_{C}$ whose intuitive meaning is that an element of $\llbracket A \rrbracket$ or $\llbracket \underline{C} \rrbracket$ approximates a closed term of type $A$ or $\underline{C}$. Given $d \in \llbracket A \rrbracket$ and a closed expression $e: A$, we define $d \triangleleft_{A} e$ by:

$$
\begin{aligned}
d \triangleleft_{\text {bool }} e & \Longleftrightarrow(d=\mathrm{tt} \wedge e=\operatorname{true}) \vee(d=\mathrm{ff} \wedge e=\text { false }) \\
d \triangleleft_{\text {nat }} e & \Longleftrightarrow d=n \wedge e=\operatorname{succ}^{n} 0 \\
d \triangleleft_{\text {unit }} e & \Longleftrightarrow d=\star \wedge e=() \\
d \triangleleft_{A \rightarrow \underline{C}} e & \Longleftrightarrow \forall d^{\prime}, e^{\prime} .\left(d^{\prime} \triangleleft_{A} e^{\prime} \Rightarrow d\left(d^{\prime}\right) \triangleleft_{\underline{C}} e e^{\prime}\right) \\
d \triangleleft_{E^{R}} e & \Longleftrightarrow d=e \\
d \triangleleft_{\underline{C}} \Rightarrow \underline{D} e & \Longleftrightarrow \forall d^{\prime}, c .\left(d^{\prime} \triangleleft_{\underline{C}} c \Rightarrow d\left(d^{\prime}\right) \triangleleft_{\underline{D}}(\text { with } e \text { handle } c)\right)
\end{aligned}
$$

Simultaneously we define when $d \in \llbracket A ! \Delta \rrbracket$ approximates a closed computation $c: A$ ! $\Delta$, where $d \triangleleft_{A} ! \Delta c$ holds when

- $d=\perp$, or

- $d=\operatorname{in}_{\mathrm{val}}\left(d^{\prime}\right), c \Downarrow \operatorname{val} e$ and $d^{\prime} \triangleleft_{A} e$, or

- $d=\operatorname{in}_{\iota \# \text { op }}\left(d^{\prime}, \kappa\right), c \Downarrow \iota \#$ op $e\left(y . c^{\prime}\right), d^{\prime} \triangleleft_{A \text { op }} e$, and if $d^{\prime \prime} \triangleleft_{B^{\text {op }}} e^{\prime}$ then $\kappa\left(d^{\prime \prime}\right) \triangleleft_{A}$ ! $\Delta c^{\prime}\left[e^{\prime \prime} / y\right]$.

The definition of $\triangleleft_{A}$ ! $\Delta$ refers to types $A^{\text {op }}$ and $B^{\text {op }}$ which are possibly larger than $A$, thus we again use [14, Theorem 4.16] to establish existence of minimal such $\triangleleft_{A}$ and $\triangleleft_{\underline{C}}$, much like for the recursive types considered in [14, Section 5].

Lemma 5.5. If $d \triangleleft_{A ! \Delta} c^{\prime}$ and $c \rightsquigarrow c^{\prime}$ then $d \triangleleft_{A} ! \Delta c$.

Proof. There is nothing to prove if $d=\perp$. The other two cases follow because $c \rightsquigarrow c^{\prime}$ and $c^{\prime} \Downarrow r$ imply $c \Downarrow r$. For instance, if $d \triangleleft_{A} ! \Delta c^{\prime}$ holds because $d=\operatorname{in}_{\mathrm{val}}\left(d^{\prime}\right), c \Downarrow$ val $e$ and $d^{\prime} \triangleleft_{A} e$ for some $d^{\prime}$ and $e$, then $d \triangleleft_{A} ! \Delta c$ holds because $c \rightsquigarrow c^{\prime}$ implies $c \Downarrow$ val $e$ and so we may reuse $d^{\prime}$ and $e$.

Lemma 5.6. The relations $\triangleleft_{A}$ and $\triangleleft_{\underline{C}}$ are closed under suprema of chains in the first argument. The relations $\triangleleft_{\underline{C}}$ relate $\perp$ to every computation.

Proof. The second statement holds by the definition of $\triangleleft_{C}$. For the first statement we proceed by induction on the type. The base cases hold because the predomains for ground types are flat. For $A \rightarrow \underline{C}$, suppose $\left(d_{n}\right)_{n}$ is a chain in $\llbracket A \rightarrow \underline{C} \rrbracket$ and $d_{n} \triangleleft_{A \rightarrow C} e$ for all $n$. Consider any $d^{\prime}, e^{\prime}$ such that $d^{\prime} \triangleleft_{A} e^{\prime}$. Then $d_{n}\left(d^{\prime}\right) \triangleleft_{\underline{C}} e e^{\prime}$ for all $n$. Suprema in $\bar{\llbracket} A \rightarrow \underline{C} \rrbracket$ are defined pointwise, so we can use the induction hypothesis for $\underline{C}$ and get

$$
\left(\bigvee_{n} d_{n}\right)\left(d^{\prime}\right)=\bigvee_{n} d_{n}\left(d^{\prime}\right) \triangleleft_{\underline{C}} e e^{\prime},
$$

hence $\bigvee_{n} d_{n} \triangleleft_{\underline{C}} e$ as desired. Handler types are treated similarly. Consider a computation type $A$ ! $\Delta$, a closed computation $c: A$ ! $\Delta$ and a chain $\left(d_{n}\right)_{n}$ in $\llbracket A$ ! $\Delta \rrbracket$ such that $d_{n}{ }^{\triangleleft} \underline{C}$ for all $n$. There are three kinds of chains in $\llbracket A ! \Delta \rrbracket$ :

- $\left(d_{n}\right)_{n}$ is constantly $\perp$ : then $\bigvee_{n} d_{n}=\perp \triangleleft_{\underline{C}} c$.

- for large enough $n$ there are $d_{n}^{\prime}$ such that $d_{n}=\operatorname{in}_{\text {val }}\left(d_{n}^{\prime}\right)$ : then $\left(d_{n}^{\prime}\right)_{n}$ form a chain in $\llbracket A \rrbracket$. Because operational semantics is deterministic, there exists a single $e$ such that $c \Downarrow$ val $e$, and $d_{n}^{\prime} \triangleleft_{A} e$. By induction hypothesis for $A$ we have $\bigvee_{n} d_{n}^{\prime} \triangleleft_{A} e$, from which $\bigvee_{n} d_{n} \triangleleft_{A}$ ! $\Delta c$ follows because $\bigvee_{n} d_{n}=\mathrm{in}_{\mathrm{val}}\left(\bigvee_{n} d_{n}^{\prime}\right)$.

- there are $\iota$ and op such that for large enough $n$ there are $d_{n}^{\prime}$ and $\kappa_{n}^{\prime}$ such that $d_{n}=$ $\operatorname{in}_{\iota \# \text { op }}\left(d_{n}^{\prime}, \kappa_{n}\right)$ : this case is treated analogously to the previous one. 
Lemma 5.7. Let $\Gamma$ be the context $x_{1}: A_{1}, \ldots, x_{n}: A_{n}$. Suppose that for each $1 \leqslant i \leqslant n$ we have $d_{i} \in \llbracket A_{i} \rrbracket$ and a closed expression $\vdash e_{i}: A_{i}$ such that $d_{i} \triangleleft A_{i} e_{i}$.

(1) If $\Gamma \vdash e: A$ then $\llbracket \Gamma \vdash e: A \rrbracket\left(d_{1}, \ldots, d_{n}\right) \triangleleft_{A} e\left[e_{1} / x_{1}, \ldots, e_{n} / x_{n}\right]$.

(2) If $\Gamma \vdash c: \underline{C}$ then $\llbracket \Gamma \vdash c: \underline{C} \rrbracket\left(d_{1}, \ldots, d_{n}\right) \triangleleft_{\underline{C}} c\left[e_{1} / x_{1}, \ldots, e_{n} / x_{n}\right]$.

Proof. We prove the statements by induction on the derivation of the typing judgments. Let $\eta=\left(d_{1}, \ldots, d_{n}\right)$ and $\sigma=\left[e_{1} / x_{1}, \ldots, e_{n} / x_{n}\right]$. We write $[\sigma, e / x]$ for the substitution $\left[e_{1} / x_{1}, \ldots, e_{n} / x_{n}, e / x\right]$. Throughout we assume that bound variables occurring in various terms do not appear in $\sigma$ :

Cases Var, True, False, Unit, Zero, Inst: these are all trivial.

Case Succ: $\Gamma \vdash(\operatorname{succ} e)$ : nat. By the induction hypothesis, we have $\llbracket e \rrbracket \eta \triangleleft_{\text {nat }} e \sigma$. Next, notice that the closed expression $e \sigma$ : nat must be $\operatorname{succ}^{k} 0$ for some $k$, hence $\llbracket e \rrbracket \eta=k$, and so

$$
\llbracket \operatorname{succ} e \rrbracket \eta=(k+1) \triangleleft_{\text {nat }} \operatorname{succ}(e \sigma)=(\operatorname{succ} e) \sigma .
$$

Case Fun: $\Gamma \vdash($ fun $x \mapsto c): A \rightarrow \underline{C}$. If $d^{\prime} \triangleleft_{A} e^{\prime}$ then by the induction hypothesis for $c$

$$
\llbracket \Gamma, x: A \vdash c \rrbracket\left(\eta, d^{\prime}\right) \triangleleft_{\underline{C}} c\left[\sigma, e^{\prime} / x\right] .
$$

Because $\llbracket \Gamma, x: A \vdash c \rrbracket\left(\eta, d^{\prime}\right)=(\llbracket \Gamma \vdash$ fun $x \mapsto c \rrbracket \eta) d^{\prime}$ and

$$
((\text { fun } x \mapsto c) \sigma) e^{\prime}=(\text { fun } x \mapsto c \sigma) e^{\prime} \rightsquigarrow c\left[\sigma, e^{\prime} / x\right],
$$

we may use Lemma 5.5 to get the desired conclusion

$$
(\llbracket \Gamma \vdash \operatorname{fun} x \mapsto c \rrbracket \eta) d^{\prime} \triangleleft_{\underline{C}}((\text { fun } x \mapsto c) \sigma) e^{\prime} .
$$

Most other cases in the proof follow the same pattern, so we shall not explicitly mention uses of Lemma 5.5 anymore.

Case Hand: $\Gamma \vdash$ (handler val $x \mapsto c_{v} \mid$ ocs $): A ! \Delta \Rightarrow B ! \Delta^{\prime}$. We abbreviate the handler as $h$. Assuming $d \triangleleft_{A} ! \Delta c$ we need to show that

$$
(\llbracket h \rrbracket \eta)(d) \triangleleft_{B ! \Delta^{\prime}}(\text { with } h \sigma \text { handle } c) .
$$

We proceed by an induction on $d$ :

- If $d=\perp$ then the conclusion follows because $\llbracket h \rrbracket \eta$ is strict.

- If $d=\operatorname{in}_{\text {val }}\left(d^{\prime}\right)$ then $d \triangleleft_{A}$ ! $\Delta c$ implies $c \Downarrow$ val $e^{\prime}$ and $d^{\prime} \triangleleft_{A} e^{\prime}$ for some $e^{\prime}$ and $d^{\prime}$. In this case, by induction hypothesis for $c_{v}$,

$$
(\llbracket h \rrbracket \eta)(d)=\llbracket \Gamma, x: A \vdash c_{v} \rrbracket\left(\eta, d^{\prime}\right) \triangleleft_{b ! \Delta} c_{v}\left[\sigma, e^{\prime} / x\right],
$$

and

$$
\text { (with } h \sigma \text { handle } c) \rightsquigarrow \cdots \rightsquigarrow\left(c_{v} \sigma\right)\left[e^{\prime} / x\right]=c_{v}\left[\sigma, e^{\prime} / x\right] \text {. }
$$

- If $d=\operatorname{in}_{\iota \# \text { op }}\left(d^{\prime}, \kappa\right)$ then $d \triangleleft_{A}$ ! $\Delta c$ implies $c \Downarrow \iota \#$ op $e^{\prime}\left(y . c^{\prime}\right), d^{\prime} \triangleleft_{A^{\text {op }}} e^{\prime}$ and $\kappa \triangleleft_{B^{\circ \mathrm{p}} \rightarrow A}$ ! $\Delta$ (fun $y \mapsto c^{\prime}$ ). Now

$$
(\llbracket h \rrbracket \eta)\left(\operatorname{in}_{\iota \# \mathrm{op}}\left(d^{\prime}, \kappa\right)\right)=\llbracket o c s \rrbracket_{\iota \# \mathrm{op}}\left(\eta, d^{\prime}, \llbracket h \rrbracket \eta \circ \kappa\right)
$$

and

$$
\text { (with } h \sigma \text { handle } c) \rightsquigarrow \cdots \rightsquigarrow(\text { ocs } \sigma)_{\iota \text { op }}\left(e^{\prime},\left(\text { fun } y \mapsto \text { with } h \sigma \text { handle } c^{\prime}\right)\right. \text { ), }
$$

therefore it suffices to prove

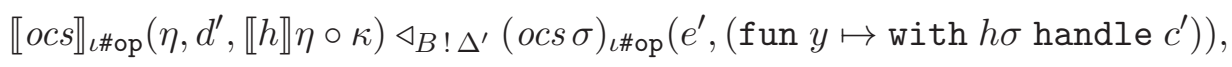

which we do by induction on the length of ocs. When ocs is nil the statement becomes

$$
\operatorname{in}_{\iota \# \text { op }}\left(d^{\prime}, \llbracket h \rrbracket \eta \circ \kappa\right) \triangleleft_{B ! \Delta^{\prime}} \iota \# \text { op } e^{\prime}\left(y \text {. with } h \sigma \text { handle } c^{\prime}\right) .
$$


We already know $d^{\prime} \triangleleft A^{\text {op }} e^{\prime}$, and still need

$$
\llbracket h \rrbracket \eta\left(\kappa\left(d^{\prime \prime}\right)\right) \triangleleft_{B ! \Delta^{\prime}}\left(\text { with } h \sigma \text { handle } c^{\prime}\left[e^{\prime \prime} / / y\right]\right)
$$

assuming $d^{\prime \prime} \triangleleft_{B_{\text {op }}} e^{\prime \prime}$. This follows from the available induction hypotheses.

When ocs is $\left(\iota^{\prime} \# \mathrm{op}^{\prime} x k \mapsto c^{\prime \prime} \mid\right.$ ocs $\left.s^{\prime}\right)$ there are two further subcases:

- if $\iota \#$ op $=\iota^{\prime} \# \mathrm{op}^{\prime}$ then we get

$$
\begin{aligned}
& \llbracket \Gamma, x, k \vdash c^{\prime \prime} \rrbracket(\eta, d, \llbracket h \rrbracket \eta \circ \kappa) \triangleleft_{B ! \Delta^{\prime}} \\
& \quad c^{\prime \prime}\left[\sigma, e^{\prime} / x,\left(\text { fun } y \mapsto \text { with } h \sigma \text { handle } c^{\prime}\right) / k\right]
\end{aligned}
$$

which holds by induction on $c^{\prime \prime}$,

- if $\iota$ \#op $\neq \iota^{\prime} \#$ op' then we are left with

$$
\begin{aligned}
& \llbracket o c s^{\prime} \rrbracket_{\iota \# \text { op }}\left(\eta, d^{\prime}, \llbracket h \rrbracket \eta \circ \kappa\right) \triangleleft_{B} ! \Delta^{\prime} \\
& \quad\left(\text { ocs } s^{\prime} \sigma\right)_{\iota \# \text { op }}\left(e,\left(\text { fun } y \mapsto \text { with } h \sigma \text { handle } c^{\prime}\right)\right),
\end{aligned}
$$

which is just the induction hypothesis for $o c s^{\prime}$.

Cases IfThenElse, Match, Absurd: We consider only

$$
\Gamma \vdash\left(\text { if } e \text { then } c_{1} \text { else } c_{2}\right): \underline{C},
$$

as MATCH is similar and ABSURD is vacuous. Because $e \sigma$ is a closed expression of type bool it is either true or false. Let us take a look at the first possibility. By induction hypothesis for $c_{1}$ we have $\llbracket c_{1} \rrbracket \eta \triangleleft_{\underline{C}} c_{1} \sigma$. Again, because 【if true then $c_{1}$ else $c_{2} \rrbracket \eta=$ $\llbracket c_{1} \rrbracket \eta$ and

$$
\text { (if true then } c_{1} \text { else } c_{2} \text { ) } \sigma \rightsquigarrow c_{1} \sigma
$$

it follows that

$$
\text { 【if true then } \left.c_{1} \text { else } c_{2} \rrbracket \eta \triangleleft_{\underline{C}} \text { (if true then } c_{1} \text { else } c_{2}\right) \sigma,
$$

as required.

Case App: $\Gamma \vdash e_{1}^{\prime} e_{2}^{\prime}: \underline{C}$ where $\Gamma \vdash e_{1}^{\prime}: A \rightarrow \underline{C}$ and $\Gamma \vdash e_{2}^{\prime}: A$. By induction hypotheses for $e_{1}^{\prime}$ and $e_{2}^{\prime}$ we have $\llbracket e_{1}^{\prime} \rrbracket \eta \triangleleft_{A} \rightarrow \underline{C} e_{1}^{\prime} \sigma$ and $\llbracket e_{2}^{\prime} \rrbracket \eta \triangleleft_{A} e_{2} \sigma$, therefore by the definition of $\triangleleft_{A} \rightarrow \underline{C}$,

$$
\llbracket e_{1}^{\prime} e_{2}^{\prime} \rrbracket \eta=\left(\llbracket e_{1}^{\prime} \rrbracket \eta\right)\left(\llbracket e_{2}^{\prime} \rrbracket \eta\right) \triangleleft_{\underline{C}}\left(e_{1}^{\prime} \sigma\right)\left(e_{2}^{\prime} \sigma\right)=\left(e_{1}^{\prime} e_{2}^{\prime}\right) \sigma .
$$

Case VAL: $\Gamma \vdash \operatorname{val} e: A$ ! $\Delta$. By induction on $e$ we have $\llbracket e \rrbracket \eta \triangleleft_{A} e \sigma$, therefore by the second clause in the definition of $\triangleleft_{A}$ ! $\Delta$

$$
\llbracket \operatorname{val} e \rrbracket \eta=\operatorname{in}_{\operatorname{val}}(\llbracket e \rrbracket \eta) \triangleleft A ! \Delta \operatorname{val}(e \sigma)=(\operatorname{val} e) \sigma .
$$

Case Op: $\Gamma \vdash \iota$ \#оp $e(y . c): A ! \Delta$. This case works much like a combination of VAL and App, so we omit the details.

Case Let: $\Gamma \vdash\left(\right.$ let $x=c_{1}$ in $\left.c_{2}\right): B ! \Delta$. This case is treated like a handler which only has a val case.

Case Wiтн: $\Gamma \vdash$ with $e$ handle $c: \underline{D}$ where $\Gamma \vdash e: \underline{C} \Rightarrow \underline{D}$ and $\Gamma \vdash c: \underline{C}$. By induction hypotheses we have $\llbracket e \rrbracket \eta \triangleleft_{\underline{C}} \Rightarrow \underline{D}$ e $e \sigma$ and $\llbracket c \rrbracket \eta \triangleleft_{\underline{C}} c \sigma$, therefore by the definition of $\triangleleft_{\underline{C}} \Rightarrow \underline{D}$

$$
(\llbracket e \rrbracket \eta)(\llbracket c \rrbracket \eta) \triangleleft_{D}(\text { with } e \sigma \text { handle } c \sigma) \text {. }
$$

The left-hand side equals $\llbracket$ with $e$ handle $c: \underline{D} \rrbracket \eta$ and the right (with $e$ handle $c$ ) $\sigma$, so we are done. 
Case LetRec: $\Gamma \vdash$ let rec $f x=c_{1}$ in $c_{2}: \underline{D}$. After a short calculation the problem reduces to showing that

$$
g \triangleleft_{A \rightarrow \underline{C}}\left(\text { fun } x \mapsto \text { let } \operatorname{rec} f x=c_{1} \sigma \text { in } c_{1} \sigma\right)
$$

where $g$ is the least fixed-point of the operator $\Phi: \llbracket A \rightarrow \underline{C} \rrbracket \rightarrow \llbracket A \rightarrow \underline{C} \rrbracket$, defined by

$$
\Phi(h)=\lambda a \in \llbracket A \rrbracket \cdot \llbracket \Gamma, f, x \vdash c_{1} \rrbracket(\eta, h, a) .
$$

By Lemma 5.6 it suffices to show that

$$
h \triangleleft_{A \rightarrow \underline{C}}\left(\text { fun } x \mapsto \text { let rec } f x=c_{1} \sigma \text { in } c_{1} \sigma\right)
$$

implies

$$
\Phi(h) \triangleleft_{A \rightarrow \underline{C}}\left(\text { fun } x \mapsto \text { let rec } f x=c_{1} \sigma \text { in } c_{1} \sigma\right) .
$$

This amounts to proving that if $d \triangleleft_{A} e$ then

$$
\begin{aligned}
\Phi(h)(d) & =\llbracket \Gamma, f, x \vdash c_{1} \rrbracket(\eta, h, d) \\
& { }_{\underline{C}} c_{1}\left[\sigma,\left(\text { fun } x \mapsto \text { let rec } f x=c_{1} \sigma \text { in } c_{1} \sigma\right) / f, e / x\right],
\end{aligned}
$$

which holds by the induction hypothesis for $c_{1}$.

Case SubExpr, SubComp: For SubExpr, take $\Gamma \vdash e: A^{\prime}$ where $\Gamma \vdash e: A$ and $A \leqslant A^{\prime}$. From induction hypothesis, we get $\llbracket \Gamma \vdash e: A \rrbracket \eta \triangleleft_{A} e \sigma$. Since $\llbracket A \rrbracket=\llbracket A^{\prime} \rrbracket$, we have $\triangleleft_{A}=\triangleleft_{A^{\prime}}$. Additionally, $\llbracket \Gamma \vdash e: A^{\prime} \rrbracket=\llbracket \Gamma \vdash e: A \rrbracket$, hence $\llbracket e \rrbracket \eta \triangleleft_{A^{\prime}} e \sigma$. For SubComp, the proof is similar.

Corollary 5.8 (Adequacy). If $\vdash c$ : unit! $\Delta$ and $\llbracket c \rrbracket=\operatorname{in}_{\mathrm{val}}(\star)$ then $c \Downarrow \operatorname{val}()$.

Proof. By the previous lemma $\llbracket c \rrbracket \triangleleft_{\text {unit } ! \Delta} c$. Therefore, if $\llbracket c \rrbracket=\mathrm{in}_{\text {val }}(\star)$ then $c \Downarrow$ val () by the definition of $\triangleleft_{\text {unit }} ! \Delta$.

The stated adequacy suffices for our purposes, but of course similar statements holds for other ground types. Regarding operations, if $\llbracket c \rrbracket=\mathrm{in}_{\iota \# \text { op }}(d, \kappa)$, then $\iota \#$ op $\in \Delta$ and so $c \Downarrow \iota \#$ op $e k$ for some $e$ and $k$ such that $\llbracket e \rrbracket=d$ and $\llbracket k \rrbracket=\kappa$. Therefore, if $\llbracket c \rrbracket \neq \perp$ then $c \Downarrow r$ for some result $r$.

\section{Equational Reasoning}

6.1. Contextual and denotational equivalence. In this section we provide principles that allow us to reason about programs. Let us first recall how contextual equivalence is defined. An expression context $\mathcal{E}$ is a computation with several occurrences of a hole [] in positions where an expression is expected. When the hole is plugged with an expression $e$ we get a computation $\mathcal{E}[e]$. A computation context $\mathcal{E}$ is defined analogously, except that the holes appear where computations are expected.

We say that expressions $e$ and $e^{\prime}$ are contextually equivalent, written $e \approx e^{\prime}$, when for all expression contexts $\mathcal{E}$ such that $\mathcal{E}[e]$ and $\mathcal{E}\left[e^{\prime}\right]$ are both of type unit! $\Delta$, we have $\mathcal{E}[e] \Downarrow \operatorname{val}()$ if and only if $\mathcal{E}\left[e^{\prime}\right] \Downarrow \operatorname{val}()$. Contextual equivalence of computations is defined analogously.

Contextually equivalent expressions or computations may be interchanged anywhere in the code. Therefore, it is quite useful to know that a certain contextual equivalence holds, but unfortunately it is difficult to work directly with contextual equivalence. Luckily, denotational equivalence is more easily handled and is related to contextual equivalence by the adequacy theorem. 
We write $e \equiv e^{\prime}$ and $c \equiv c^{\prime}$ when the denotations of two expressions or computations are the same. More precisely, if $\Gamma \vdash e: A$ and $\Gamma \vdash e^{\prime}: A$ then $\Gamma \vdash e \equiv e^{\prime}: A$, or just $e \equiv e^{\prime}$, means $\llbracket \Gamma \vdash e: A \rrbracket=\llbracket \Gamma \vdash e^{\prime}: A \rrbracket$, and similarly for computations.

Proposition 6.1. Denotationally equal expressions are contextually equivalent, and likewise for computations.

Proof. Suppose $e \equiv e^{\prime}$ and consider an expression context $\mathcal{E}$ such that $\mathcal{E}[e]$ and $\mathcal{E}\left[e^{\prime}\right]$ both have type unit! $\Delta$. Assume $\mathcal{E}[e] \Downarrow \operatorname{val}()$. By soundness of denotational semantics $\llbracket \mathcal{E}[e] \rrbracket=$ $\operatorname{in}_{\text {val }}(\star)$. By assumption $\llbracket e \rrbracket=\llbracket e^{\prime} \rrbracket$ and because denotational semantics is compositional it follows that $\llbracket \mathcal{E}[e] \rrbracket=\llbracket \mathcal{E}\left[e^{\prime}\right] \rrbracket$. Now by adequacy $\mathcal{E}\left[e^{\prime}\right] \Downarrow$ val (). The proof for computations is the same.

Denotational equivalence is a congruence and is preserved by well-typed substitutions. It validates the following $\beta$-rules, where the various expressions and computations have suitable types, and $h$ stands for handler val $x \mapsto c_{v} \mid$ ocs:

$$
\begin{aligned}
& \text { if true then } c_{1} \text { else } c_{2} \equiv c_{1} \\
& \text { if false then } c_{1} \text { else } c_{2} \equiv c_{2} \\
& \text { match } 0 \text { with } 0 \mapsto c_{1} \mid \operatorname{succ} x \mapsto c_{2} \equiv c_{1} \\
& \operatorname{match}(\operatorname{succ} e) \text { with } 0 \mapsto c_{1} \mid \operatorname{succ} x \mapsto c_{2} \equiv c_{2}[e / x] \\
& \text { (fun } x \mapsto c) e \equiv c[e / x] \\
& \text { let } x=\operatorname{val} e \text { in } c \equiv c[e / x] \\
& \text { let } x=e_{1} \# \text { op } e_{2}\left(y . c_{1}\right) \text { in } c_{2} \equiv e_{1} \# \text { op } e_{2}\left(y \text {. let } x=c_{1} \text { in } c_{2}\right) \\
& \text { let rec } f x=c_{1} \text { in } c_{2} \equiv c_{2}\left[\left(\text { fun } x \mapsto \text { let rec } f x=c_{1} \text { in } c_{1}\right) / f\right] \\
& \text { with } h \text { handle }(\operatorname{val} e) \equiv c_{v}[e / x] \\
& \text { with } h \text { handle }(\iota \# \text { op } e(y . c)) \equiv o c s_{\iota \# \text { op }}(e,(\text { fun } y \mapsto \text { with } h \text { handle } c))
\end{aligned}
$$

We also have the following $\eta$-rules, provided the expressions and computations have easily guessed types:

$$
\begin{aligned}
e & \equiv() \\
\text { fun } x \mapsto e x & \equiv e \\
\text { let } x=c \text { in val } x & \equiv c \\
\text { if } e \text { then } c[\text { true } / x] \text { else } c[\text { false } / x] & \equiv c[e / x] \\
\text { match } e \text { with } 0 \mapsto c[0 / x] \mid \operatorname{succ} y \mapsto c[\operatorname{succ} y / x] & \equiv c[e / x] \\
\text { absurd } e & \equiv c[e / x]
\end{aligned}
$$

We omit the proofs because they just involve unfolding of semantic definitions. An exception is the $\eta$-rule for let binding, which is proved by induction in the next section.

A variety of other equivalences is readily validated, for example

$$
\text { let } x=c_{1} \text { in } c_{2} \equiv \text { with (handler val } x \mapsto c_{2} \mid \text { nil) handle } c_{1}
$$

and the "associativity" of let binding 12

$$
\text { let } x=\left(\text { let } y=c_{1} \text { in } c_{2}\right) \text { in } c_{3} \equiv \text { let } y=c_{1} \text { in }\left(\text { let } x=c_{2} \text { in } c_{3}\right) \text {, }
$$

where $y$ must not occur freely in $c_{3}$, see [20, 21] for other examples. 
6.2. An induction principle for effects. The induction principle for the computation domains from Section 5.1 is useful for deriving general laws that do not depend on a particular choice of effects. It is less useful for specific examples which involve a carefully chosen set of effects and handlers, because it forces us to consider operations that have nothing to do with the situation at hand. The induction principle Theorem 5.2 remedies the drawback.

A typical application arises when we prove an equivalence of the form $\mathcal{E}[c] \equiv \mathcal{E}^{\prime}[c]: \underline{C}$ for all computations $c$ of a suitable type $A$ ! $\Delta$. The computation contexts $\mathcal{E}$ and $\mathcal{E}^{\prime}$ are interpreted as continuous maps $\llbracket \mathcal{E} \rrbracket, \llbracket \mathcal{E}^{\prime} \rrbracket: \llbracket A ! \Delta \rrbracket \rightarrow \llbracket \underline{C} \rrbracket$, and the equivalence may be phrased as

$$
\forall t \in \llbracket A ! \Delta \rrbracket \cdot \llbracket \mathcal{E} \rrbracket(t)=\llbracket \mathcal{E}^{\prime} \rrbracket(t) .
$$

The equality inside the quantifier is an admissible predicate on $\llbracket A ! \Delta \rrbracket$, so the induction principle applies. It is a bit cumbersome to perform the proof using the semantic brackets 【-】 all over the place. Instead, with a bit of flexibility in notation, we can write the proof in a syntactic manner as follows:

(1) We write $\perp$ for a non-terminating computation, e.g., let rec $f x=f x$ in $f($ ), and check that $\mathcal{E}[\perp] \equiv \mathcal{E}^{\prime}[\perp]$.

(2) We verify that $\mathcal{E}[\operatorname{val} e] \equiv \mathcal{E}^{\prime}[\operatorname{val} e]$ where $e$ is a meta-variable of type $A$.

(3) We verify, for each $\iota \#$ op $\in \Delta$,

$$
\mathcal{E}[\iota \# \text { op } e(y . \kappa y)] \equiv \mathcal{E}^{\prime}[\iota \# \text { op } e(y . \kappa y)]
$$

where $e$ is a meta-variable of type $A^{\text {op }}$ and $\kappa$ is a meta-variable of type $B^{\text {op }} \rightarrow A ! \Delta$. The induction hypothesis is that $\mathcal{E}\left[\kappa e^{\prime}\right] \equiv \mathcal{E}^{\prime}\left[\kappa e^{\prime}\right]$ for all expressions $e^{\prime}$ of type $B^{\text {op }}$.

We apply the method to prove the $\eta$-rule

$$
\text { let } x=c \text { in val } x \equiv c
$$

by induction:

(1) (let $x=\perp$ in val $x) \equiv \perp$ because let is strict in both arguments,

(2) $($ let $x=(\operatorname{val} a)$ in val $x) \equiv(\operatorname{val} x)[a / x] \equiv$ val $a$ by a $\beta$-rule for let,

(3) The induction step is proved by

$$
\text { let } \begin{aligned}
x & =(\iota \# \text { op } e(y \cdot \kappa y)) \text { in val } x \\
& \equiv \iota \text { op } e(y \cdot \text { let } x=\kappa y \text { in } \operatorname{val} x) \\
& \equiv \iota \text { ८op } e(y \cdot \kappa y)
\end{aligned}
$$

where we used a $\beta$-rule in the first step and the induction hypothesis for $\kappa y$ in the second.

A non-trivial application of the induction principle is presented in Section 7.2 ,

\section{EXAmple: mutable References}

As a more elaborate example we consider mutable references. In core Eff they are implemented by the effect ref with operations

$$
\text { lookup : unit } \rightarrow A \text { and update }: A \rightarrow \text { unit, }
$$


where $A$ is a fixed pure type (in full Eff we could use a parameter). The handler which handles the reference given by an expression $r: \operatorname{ref}^{R}$ is defined by (the underscore indicates an ignored parameter):

$$
\begin{aligned}
& \text { state }_{r} \stackrel{\text { def }}{=} \text { handler val } x \mapsto \operatorname{val}(\text { fun } s \mapsto \operatorname{val} x) \\
& \mid r \# \text { lookup }-k \mapsto \operatorname{val}(\text { fun } s \mapsto \text { let } f=k s \text { in } f s) \\
& \mid r \# \text { update } s^{\prime} k \mapsto \operatorname{val}\left(\text { fun } s \mapsto \text { let } f=k() \text { in } f s^{\prime}\right)
\end{aligned}
$$

This is just the usual monadic-style treatment of state which wraps a computation into a state-carrying function. For any instance $\iota \in \mathcal{I}_{\text {ref }}$, pure type $B$, and $\operatorname{dirt} \Delta$, the computation state $_{\iota}$ has the type

$$
B !(\{\iota \# \text { lookup, ı\#update }\} \cup \Delta) \Rightarrow(A \rightarrow B ! \Delta) ! \Delta .
$$

The type says that the handler erases lookups and updates of $\iota$ from a computation. The return type $(A \rightarrow B ! \Delta) ! \Delta$ has an outer dirt $\Delta$ because effects could happen before the first lookup or update. Note that the double dirt would not arise if we took the call-bypush-value approach to handlers [19].

The handler wraps a handled computation $c$ of type $B ! \Delta$ into a function expecting the current state, which explains the type $A \rightarrow B ! \Delta$. In practice such a function is immediately applied to an initial state $e$ of type $A$ (such a final transformation of handled computations is so common that in full Eff handlers have a special finally case just for this purpose):

$$
\text { let } f=(\text { with } h \text { handle } c) \text { in } f e
$$

The type of this computation is simply $B ! \Delta$. In particular, if the only effects in $c$ are lookups and updates of $\iota$, we get a pure computation.

If the type of $r$ is weaker, for example $r e f^{\left\{\iota_{1}, \iota_{2}\right\}}$, we are not able to deduce anything useful. The best we can do is

$$
\text { state }_{r}: B ! \Delta \Rightarrow(A \rightarrow B ! \Delta) ! \Delta
$$

for any dirt $\Delta$.

We may handle several references at once by wrapping a computation into several handlers. For example, let $c$ be the computation which swaps the contents of two references:

$$
\begin{aligned}
& \text { let } y_{1}=\iota_{1} \# \text { lookup }() \text { in } \\
& \text { let } y_{2}=\iota_{2} \# \text { lookup }() \text { in } \\
& \text { let }-=\iota_{1} \# \text { update } y_{2} \text { in } \\
& \text { let }-=\iota_{2} \# \text { update } y_{1} \text { in }(\operatorname{val}())
\end{aligned}
$$

By itself, $c$ has the type

$$
\text { unit ! }\left\{\iota_{1} \# \text { lookup, } \iota_{1} \# \text { update, } \iota_{2} \# \text { lookup, } \iota_{2} \# \text { update }\right\} \text {, }
$$

When $c$ is wrapped by the handler $h_{2}=$ state $_{\iota_{2}}$,

$$
\text { let } f_{2}=\left(\text { with } h_{2} \text { handle } c\right) \text { in } f_{2} e_{2} \text {, }
$$

the type becomes unit! $\left\{\iota_{1} \#\right.$ lookup, $\iota_{1} \#$ update $\}$. When the handler $h_{1}=$ state $_{\iota_{1}}$ is used on top of that,

$$
\text { let } f_{1}=\left(\text { with } h_{1} \text { handle }\left(\text { let } f_{2}=\left(\text { with } h_{2} \text { handle } c\right) \text { in } f_{2} e_{2}\right)\right) \text { in } f_{1} e_{1}
$$


we get the pure type unit! $\varnothing$. Beware, it is important that the state is initialized at the correct point in the computation. For instance,

$$
\text { let } f_{1}=\left(\text { with } h_{1} \text { handle }\left(\text { with } h_{2} \text { handle } c\right)\right) \text { in }\left(\text { let } f_{2}=f_{1} e_{1} \text { in } f_{2} e_{2}\right)
$$

does not do the right thing. We are warned about possible trouble by the effect system which gives the computation the type unit! $\left\{\iota_{1} \#\right.$ lookup, $\iota_{1} \#$ update $\}$ - the operations for $\iota_{1}$ are escaping the handlers! A less modular way of handling two instances is to create a new handler with four operation cases, two for each of the instances.

7.1. Reasoning about references. With handlers the workings of a computation may be inspected in a highly intensional way. Consequently, there are few generally valid observational equivalences. However, when known handlers are used to handle operations, we may derive equivalences that describe the behavior of operations. The situation is opposite to that of [19], where we start with an equational theory for operations and require that the handlers respect it.

We demonstrate the technique for mutable state. Let $h=$ state $_{\iota}$ and abbreviate

$$
\text { let } f=(\text { with } h \text { handle } c) \text { in } f e
$$

as $\mathcal{H}[c, e]$. Straightforward calculations give us the equivalences

$$
\begin{aligned}
\mathcal{H}[(\text { (\#lookup }()(y . c)), e] & \equiv \mathcal{H}[c[e / y], e] \\
\mathcal{H}\left[\left(\text { ı\#update } e^{\prime}(-. c)\right), e\right] & \equiv \mathcal{H}\left[c, e^{\prime}\right] \\
\mathcal{H}\left[\operatorname{val} e^{\prime}, e\right] & \equiv \operatorname{val} e^{\prime},
\end{aligned}
$$

for instance,

$$
\begin{aligned}
& \mathcal{H}\left[\left(\iota \# \text { update } e^{\prime}(-. c)\right), e\right] \\
& \equiv \text { let } f=\operatorname{val}\left(\text { fun } s \mapsto \text { let } f^{\prime}=\left(\text { fun }_{-} \mapsto \text { with } h \text { handle } c\right)\left(\text { ) in } f^{\prime} e^{\prime}\right) \text { in } f e\right. \\
& \equiv \text { let } f=\operatorname{val}\left(\text { fun } s \mapsto \mathcal{H}\left[c, e^{\prime}\right]\right) \text { in } f e \\
& \equiv\left(\text { fun } s \mapsto \mathcal{H}\left[c, e^{\prime}\right]\right) e \\
& \equiv \mathcal{H}\left[c, e^{\prime}\right] \text {. }
\end{aligned}
$$

These suffice for simple equational reasoning about state. If we read them as rewrite rules they allow us to progressively transform a computation to a simpler form. In fact, the transformations mimic the usual coalgebraic operational semantics for state [18].

Of course, a realistic computation will contain several handlers. As long as they do not interfere with each other, we can still use equivalences to usefully manipulate them. For example, if $h^{\prime}$ is a handler with no operation case for $\iota \#$ lookup then

$$
\begin{aligned}
\mathcal{H}\left[\left(\text { with } h^{\prime} \text { handle } \iota \# \text { lookup () }(y \cdot c)\right), e\right] \\
\quad \equiv \mathcal{H}\left[\left(\text { llookup }()\left(y \cdot \text { with } h^{\prime} \text { handle } c\right)\right), e\right] \\
\equiv \mathcal{H}\left[\left(\text { with } h^{\prime} \text { handle } c[e / y]\right), e\right] .
\end{aligned}
$$

It may happen that a lookup or an update is nested deeply inside several handlers. The above transformation allows us to hoist the operation out of the inner handlers so that it is handled by the outer handler, as long as the inner handlers do not attempt to handle $\iota$. The transformation applies to let bindings too, as they are like handlers without operation cases. 
We may validate the seven standard equations governing state [16]. There are four combinations of lookup and update (in the first equation $y$ does not occur free in $c$ ):

$$
\begin{aligned}
& \left.\mathcal{H}\left[\iota \# \text { lookup () (y.ı\#update } y\left(\_. c\right)\right), e\right] \equiv \mathcal{H}[c, e] \\
& \mathcal{H}[\iota \# \text { lookup () (y.ı\#lookup () (z.c)),e] } \equiv \mathcal{H}[\iota \# \text { lookup () }(y . c[y / z]), e] \\
& \mathcal{H}\left[\iota \# \text { update } e\left(\ldots \text {. } \iota \text { \#update } e^{\prime}\left({ }_{-} . c\right)\right), e\right] \equiv \mathcal{H}\left[\iota \text { \#update } e^{\prime}\left({ }_{-} . c\right), e\right] \\
& \mathcal{H}\left[\iota \# \text { update } e \left(\_. \iota \# \text { lookup () (y.c)),e] } \equiv \mathcal{H}\left[\iota \text { \#update } e\left(\_. c[e / y]\right), e\right]\right.\right.
\end{aligned}
$$

For instance, the first equation is validated as follows:

$$
\begin{aligned}
\mathcal{H}[\iota \# \text { lookup }()(y . \iota \# \text { update } y(\ldots . c)), e] & \\
& \equiv \mathcal{H}[\iota \text { \#update } e(-c), e] \\
& \equiv \mathcal{H}[c, e]
\end{aligned}
$$

Three more equations describe commutativity of lookups and updates at different distances. Let $\iota_{1} \neq \iota_{2}$, and write $\mathcal{H}_{1}$ and $\mathcal{H}_{2}$ for the the abbreviation $\mathcal{H}$ with respect to $\iota_{1}$ and $\iota_{2}$, respectively:

$$
\begin{aligned}
& \mathcal{H}_{1}\left[\mathcal{H}_{2}\left[\iota_{1} \# \text { lookup () }\left(y_{1} . \iota_{2} \# \text { lookup () }\left(y_{2} . c\right)\right), e_{2}\right], e_{1}\right] \\
& \equiv \mathcal{H}_{1}\left[\mathcal{H}_{2}\left[\iota_{2} \# \text { lookup () }\left(y_{2} . \iota_{1} \# \text { Iookup () }\left(y_{1} . c\right)\right), e_{2}\right], e_{1}\right] \\
& \mathcal{H}_{1}\left[\mathcal{H}_{2}\left[\iota_{1} \text { \#update } e_{1}\left(\ldots . \iota_{2} \# \text { update } e_{2}(\ldots . c)\right), e_{2}\right], e_{1}\right] \\
& \equiv \mathcal{H}_{1}\left[\mathcal{H}_{2}\left[\iota_{2} \# \text { update } e_{2}\left(\ldots . \iota_{1} \# \text { update } e_{1}(\ldots . c)\right), e_{2}\right], e_{1}\right] \\
& \mathcal{H}_{1}\left[\mathcal{H}_{2}\left[\iota_{1} \# \text { update } e\left(\_. \iota_{2} \# \text { lookup () }\left(y_{2} . c\right)\right), e_{2}\right], e_{1}\right] \\
& \left.\equiv \mathcal{H}_{1}\left[\mathcal{H}_{2}\left[\iota_{2} \# \text { lookup () ( } y_{2} \cdot \iota_{1} \# \text { update } e(\ldots . c)\right), e_{2}\right], e_{1}\right]
\end{aligned}
$$

Let us check the last equation. The left-hand side transforms as

$$
\begin{aligned}
& \mathcal{H}_{1}\left[\mathcal{H}_{2}\left[\iota_{1} \text { \#update } e\left(\ldots \cdot \iota_{2} \# \text { lookup () }\left(y_{2} \cdot c\right)\right), e_{2}\right], e_{1}\right] \\
& \equiv \mathcal{H}_{1}\left[\iota_{1} \text { \#update } e\left(-\cdot \mathcal{H}_{2}\left[\iota_{2} \# \text { lookup }()\left(y_{2} \cdot c\right), e_{2}\right]\right), e_{1}\right] \\
& \equiv \mathcal{H}_{1}\left[\mathcal{H}_{2}\left[\iota_{2} \# \text { lookup }()\left(y_{2} \cdot c\right), e_{2}\right], e\right] \\
& \equiv \mathcal{H}_{1}\left[\mathcal{H}_{2}\left[c\left[e_{2} / y_{2}\right], e_{2}\right], e\right]
\end{aligned}
$$

and the right-hand side as

$$
\begin{aligned}
\mathcal{H}_{1}\left[\mathcal{H}_{2}\left[\iota_{2} \# \text { Iookup () }\left(y_{2} \cdot \iota_{1} \text { \#update } e(-. c)\right), e_{2}\right], e_{1}\right] \\
\quad \equiv \mathcal{H}_{1}\left[\mathcal{H}_{2}\left[\iota_{1} \# \text { update } e\left(-. c\left[e_{2} / y_{2}\right]\right), e_{2}\right], e_{1}\right] \\
\quad \equiv \mathcal{H}_{1}\left[\iota_{1} \# \text { update } e\left(-\cdot \mathcal{H}_{2}\left[c\left[e_{2} / y_{2}\right], e_{2}\right]\right), e_{1}\right] \\
\quad \equiv \mathcal{H}_{1}\left[\mathcal{H}_{2}\left[c\left[e_{2} / y_{2}\right], e_{2}\right], e\right] .
\end{aligned}
$$

The remaining two equations are proved much the same way. The symmetry in the equations also shows that it does not matter in which order we nest the handlers for $\iota_{1}$ and $\iota_{2}$.

7.2. Commutativity of non-interfering computations. Swapping lookups and updates that act on different instances is only a basic reasoning step. In practice we want to swap whole computations, as long as they do not interfere. To make the idea precise, let $\Delta_{1}=\left\{\iota_{1} \#\right.$ lookup, $\iota_{1} \#$ update $\}, \Delta_{2}=\left\{\iota_{2} \#\right.$ lookup, $\iota_{2} \#$ update $\}$, let $c_{1}$ and $c_{2}$ be computations 
of types $A_{1} ! \Delta_{1}$ and $A_{2} ! \Delta_{2}$, respectively, and $c$ a computation of type $\underline{C}$ in the context $x_{1}: A_{1}, x_{2}: A_{2}$. We would like to show the equivalence

$$
\begin{aligned}
& \mathcal{H}_{1}\left[\mathcal{H}_{2}\left[\text { let } x_{1}=c_{1} \text { in }\left(\text { let } x_{2}=c_{2} \text { in } c\right), e_{2}\right], e_{1}\right] \\
& \equiv \mathcal{H}_{1}\left[\mathcal{H}_{2}\left[\text { let } x_{2}=c_{2} \text { in }\left(\text { let } x_{1}=c_{1} \text { in } c\right), e_{2}\right], e_{1}\right] .
\end{aligned}
$$

This is a commutativity law which allows us to transpose, or run in parallel, any computations that use only non-interfering references.

First, let us establish a simpler equivalence, which we are going to use in the proof:

$$
\begin{aligned}
\mathcal{H}_{1}\left[\mathcal{H}_{2}\left[\iota_{1} \# \text { lookup () }\left(y_{1} \text {. let } x_{2}=c_{2} \text { in }\left(\text { let } x_{1}=c_{1}^{\prime} \text { in } c\right)\right), e_{2}\right], e_{1}\right] \\
\quad \equiv \mathcal{H}_{1}\left[\mathcal{H}_{2}\left[\text { let } x_{2}=c_{2} \text { in }\left(\text { let } x_{1}=\iota_{1} \# \text { lookup }()\left(y_{1} . c_{1}^{\prime}\right) \text { in } c\right), e_{2}\right], e_{1}\right]
\end{aligned}
$$

We proceed by induction on $c_{2}$. Since handlers and let are strict, both sides are $\perp$ when we set $c_{2}$ to $\perp$. Next, if $c_{2}$ is val $e_{2}$, the two sides are equal already without handlers:

$$
\begin{aligned}
& \iota_{1} \# \text { lookup () }\left(y_{1} \text {. let } x_{2}=\operatorname{val} e_{2} \text { in }\left(\text { let } x_{1}=c_{1}^{\prime} \text { in } c\right)\right) \\
& \quad \equiv \iota_{1} \# \text { lookup () }\left(y_{1} \text {. let } x_{1}=c_{1}^{\prime} \text { in } c\left[e_{2} / x_{2}\right]\right) \\
& \quad \equiv \iota_{1} \# \text { lookup () }\left(y_{1} \text {. let } x_{1}=c_{1}^{\prime} \text { in }\left(\text { let } x_{2}=\operatorname{val} e_{2} \text { in } c\right)\right) \\
& \quad \equiv \operatorname{let} x_{1}=\iota_{1} \# \text { lookup () }\left(y_{1} \cdot c_{1}^{\prime}\right) \text { in }\left(\text { let } x_{2}=\operatorname{val} e_{2} \text { in } c\right)
\end{aligned}
$$

For the induction step, suppose $c_{2}$ is a call of $\iota_{2}$ \#update (the case lookup is similar):

$$
\begin{aligned}
\mathcal{H}_{1} & {\left[\mathcal{H}_{2}\left[\iota_{1} \# \text { lookup () }\left(y_{1} \text {. let } x_{2}=\iota_{2} \# \text { update } e_{2}^{\prime}(z . \kappa z) \text { in }\left(\text { let } x_{1}=c_{1}^{\prime} \text { in } c\right)\right), e_{2}\right], e_{1}\right] } \\
& \equiv \mathcal{H}_{1}\left[\iota_{1} \# \text { lookup }()\left(y_{1} \cdot \mathcal{H}_{2}\left[\text { let } x_{2}=\kappa() \text { in }\left(\text { let } x_{1}=c_{1}^{\prime} \text { in } c\right), e_{2}^{\prime}\right]\right), e_{1}\right] \\
& \equiv \mathcal{H}_{1}\left[\mathcal{H}_{2}\left[\iota_{1} \# \text { lookup () }\left(y_{1} \text {. let } x_{2}=\kappa() \text { in }\left(\text { let } x_{1}=c_{1}^{\prime} \text { in } c\right)\right), e_{2}^{\prime}\right], e_{1}\right] \\
& \equiv \mathcal{H}_{1}\left[\mathcal{H}_{2}\left[\text { let } x_{2}=\kappa() \text { in }\left(\text { let } x_{1}=\iota_{1} \# \text { lookup }()\left(y_{1} . c_{1}^{\prime}\right) \text { in } c\right), e_{2}^{\prime}\right], e_{1}\right] \\
& \equiv \mathcal{H}_{1}\left[\mathcal{H}_{2}\left[\text { let } x_{2}=\iota_{2} \# \text { update } e_{2}^{\prime}(z . \kappa z) \text { in }\left(\text { let } x_{1}=\iota_{1} \# \text { lookup () }\left(y_{1} . c_{1}^{\prime}\right) \text { in } c\right), e_{2}\right], e_{1}\right] .
\end{aligned}
$$

In the third step we used the induction hypothesis for $\kappa()$.

We now prove the main statement by induction on $c_{1}$. When $c_{1}$ is $\perp$ or a value, the statement is easy. If $c_{1}$ is a call of $\iota_{1} \#$ lookup, we have:

$$
\begin{aligned}
\mathcal{H}_{1}\left[\mathcal{H}_{2}\right. & {\left.\left[\text { let } x_{1}=\iota_{1} \# \text { lookup () }\left(y_{1} . \kappa y_{1}\right) \text { in }\left(\text { let } x_{2}=c_{2} \text { in } c\right), e_{2}\right], e_{1}\right] } \\
& \equiv \mathcal{H}_{1}\left[\iota_{1} \# \text { lookup () }\left(y_{1} . \mathcal{H}_{2}\left[\text { let } x_{1}=\kappa y_{1} \text { in }\left(\text { let } x_{2}=c_{2} \text { in } c\right), e_{2}\right)\right], e_{1}\right] \\
& \equiv \mathcal{H}_{1}\left[\mathcal{H}_{2}\left[\text { let } x_{1}=\kappa e_{1} \text { in }\left(\text { let } x_{2}=c_{2} \text { in } c\right), e_{2}\right], e_{1}\right] \\
& \equiv \mathcal{H}_{1}\left[\mathcal{H}_{2}\left[\text { let } x_{2}=c_{2} \text { in }\left(\operatorname{let} x_{1}=\kappa e_{1} \text { in } c\right), e_{2}\right], e_{1}\right]
\end{aligned}
$$

where in the last step, we used the induction hypothesis for $\kappa e_{1}$. The last line is equivalent to the other side of the desired equivalence:

$$
\begin{aligned}
\mathcal{H}_{1}\left[\mathcal{H}_{2}\left[\text { let } x_{2}=c_{2} \text { in }\left(\text { let } x_{1}=\kappa e_{1} \text { in } c\right), e_{2}\right], e_{1}\right] \\
\quad \equiv \mathcal{H}_{1}\left[\iota_{1} \# \text { lookup () }\left(y_{1} \cdot \mathcal{H}_{2}\left[\text { let } x_{2}=c_{2} \text { in }\left(\text { let } x_{1}=\kappa y_{1} \text { in } c\right), e_{2}\right]\right), e_{1}\right] \\
\equiv \mathcal{H}_{1}\left[\mathcal{H}_{2}\left[\text { let } x_{2}=c_{2} \text { in }\left(\text { let } x_{1}=\iota_{1} \# \text { lookup }()\left(y_{1} . \kappa y_{1}\right) \text { in } c\right), e_{2}\right], e_{1}\right]
\end{aligned}
$$

The proof for update is similar. 


\section{Formalization in TwelF}

We formalized core Eff, the effect system and the safety theorems in Twelf. The files are enclosed with this paper, or can be found at the GitHub repository [4]. The code is compatible with Twelf version 1.7.1. Further instructions and description of the code can be found in the file README.md.

\section{Discussion}

The only essential feature of Eff that is missing from core Eff is dynamic creation of instances with the new $E$ construct. We omitted it because it leads to significant complications, both in the effect system and in semantics. One possible treatment of new would be to upgrade the current setup with nominal logic and nominal domain theory.

Non-termination is a computational effect which is not reflected in our effect system. It would be interesting to add a "divergence" effect. Such an effect would originate from applications of recursive functions. However, since divergence cannot be handled it would never disappear from effect information, and would likely become an uninformative nuisance. A potential remedy would be to separate general recursive definitions, which may diverge, from structural recursive definitions, which always terminate.

A realistic implementation of our effect system would only be useful if it actually inferred computational effects. Such a possibility was explored by the second author [22, and an early prototype is available in the latest implementation of Eff [5].

Acknowledgment. We thank the anonymous referees for useful suggestions and a lesson in domain theory.

\section{REFERENCES}

[1] Roberto M. Amadio and Pierre-Louis Curien. Domains and Lambda-calculi. Cambridge University Press, New York, NY, USA, 1998.

[2] Andrej Bauer and Matija Pretnar. Programming with algebraic effects and handlers. arXiv:1203.1539, 2012.

[3] Andrej Bauer and Matija Pretnar. An effect system for algebraic effects and handlers. In Reiko Heckel and Stefan Milius, editors, Algebra and Coalgebra in Computer Science, volume 8089 of Lecture Notes in Computer Science, pages 1-16. Springer Berlin Heidelberg, 2013.

[4] Andrej Bauer and Matija Pretnar. Formalization of Eff in Twelf, 2013. https://github.com/matijapretnar/twelf-eff.

[5] Andrej Bauer and Matija Pretnar. Eff, 2014. https://github.com/matijapretnar/twelf-eff

[6] You-Chin Fuh and Prateek Mishra. Type inference with subtypes. Theoretical computer science, 73(2):155-175, 1990.

[7] Ohad Kammar, Sam Lindley, and Nicolas Oury. Handlers in action. In Proceedings of the 18th ACM SIGPLAN International Conference on Functional Programming, ICFP '13, pages 145-158, New York, NY, USA, 2013. ACM.

[8] Ohad Kammar and Gordon D. Plotkin. Algebraic foundations for effect-dependent optimisations. In John Field and Michael Hicks, editors, POPL, pages 349-360. ACM, 2012.

[9] Paul Blain Levy. Call-by-push-value: Decomposing call-by-value and call-by-name. Higher-Order and Symbolic Computation, 19(4):377-414, 2006.

[10] Paul Blain Levy, John Power, and Hayo Thielecke. Modelling environments in call-by-value programming languages. Information and Computation, 185:182-210, September 2003. 
[11] J. M. Lucassen and D. K. Gifford. Polymorphic effect systems. In Proceedings of the 15th ACM SIGPLAN-SIGACT symposium on Principles of programming languages, POPL'88, pages 47-57, New York, NY, USA, 1988. ACM.

[12] Eugenio Moggi. Notions of computation and monads. Information and Computation, 93(1):55-92, 1991.

[13] Frank Pfenning and Carsten Schürmann. System description: Twelf - a meta-logical framework for deductive systems. In Harald Ganzinger, editor, CADE-16, 16th International Conference on Automated Deduction, Trento, Italy, July 7-10, 1999, Proceedings, volume 1632 of Lecture Notes in Computer Science, pages 202-206. Springer, 1999.

[14] Andrew M. Pitts. Relational properties of domains. Information and Computation, 127(2):66-90, 1996.

[15] Gordon Plotkin and John Power. Adequacy for algebraic effects. In Furio Honsell and Marino Miculan, editors, Foundations of Software Science and Computation Structures, volume 2030 of Lecture Notes in Computer Science, pages 1-24. Springer Berlin Heidelberg, 2001.

[16] Gordon Plotkin and John Power. Notions of computation determine monads. In 5th International Conference on Foundations of Software Science and Computation Structures, volume 2303 of Lecture Notes in Computer Science, pages 342-356, 2002.

[17] Gordon Plotkin and John Power. Algebraic operations and generic effects. Applied Categorical Structures, 11(1):69-94, 2003.

[18] Gordon Plotkin and John Power. Tensors of comodels and models for operational semantics. In Andrej Bauer and Michael Mislove, editors, Proceedings of the 24th Conference on the Mathematical Foundations of Programming Semantics (MFPS XXIV), volume 218 of Electronic Notes in Theoretical Computer Science, pages 295-311, 2008.

[19] Gordon D. Plotkin and Matija Pretnar. Handling algebraic effects. Logical Methods in Computer Science, 9(4), 2013.

[20] Gordon David Plotkin and Matija Pretnar. A logic for algebraic effects. In Proceedings of the TwentyThird Annual IEEE Symposium on Logic in Computer Science, LICS 2008, 24-27 June 2008, Pittsburgh, PA, USA, pages 118-129. IEEE Computer Society, 2008.

[21] Matija Pretnar. The Logic and Handling of Algebraic Effects. PhD thesis, School of Informatics, University of Edinburgh, 2010.

[22] Matija Pretnar. Inferring algebraic effects. Logical Methods in Computer Science, 10(3), 2014.

[23] John Reynolds. The meaning of types - from intrinsic to extrinsic semantics. Technical report, Department of Computer Science, University of Aarhus, 2000.

[24] Jean-Pierre Talpin and Pierre Jouvelot. Polymorphic type, region and effect inference. Journal of functional programming, 2(3):245-271, 1992.

[25] Philip Wadler. The marriage of effects and monads. ACM SIGPLAN Notices, 34(1):63-74, 1999.

[26] Keith Wansbrough and Simon Peyton Jones. Once upon a polymorphic type. In Proceedings of the 26th ACM SIGPLAN-SIGACT Symposium on Principles of Programming Languages, POPL '99, pages 15-28, New York, NY, USA, 1999. ACM.

This work is licensed under the Creative Commons Attribution-NoDerivs License. To view a copy of this license, visit http://creativecommons.org/licenses/by-nd/2.0/ or send a letter to Creative Commons, 171 Second St, Suite 300, San Francisco, CA 94105, USA, or Eisenacher Strasse 2, 10777 Berlin, Germany 